\title{
On the Distribution of Life Annuities with Stochastic Interest Rates
}

\author{
Tom Hoedemakers ${ }^{* \dagger \ddagger}$ Grzegorz Darkiewicz ${ }^{\dagger}$ Marc Goovaerts $^{\dagger \S}$
}

\begin{abstract}
In the traditional approach to life contingencies only decrements are assumed to be stochastic. In this contribution we consider the distribution of a life annuity (and a portfolio of life annuities) when also the stochastic nature of interest rates is taken into account. Although the literature concerning this topic is already quite rich, the authors usually restrict themselves to the computation of the first two or three moments. However, if one wants to determine e.g. capital requirements using more sofisticated risk measures like Value-at-Risk or Tail Value-at-Risk, more detailed knowledge about underlying distributions is required. For this purpose, we propose to use the theory of comonotonic risks developed in Dhaene et al. (2002a and 2002b), which has to be slightly adjusted to the case of scalar products. This methodology allows to obtain reliable approximations of the underlying distribution functions, in particular very accurate estimates of upper quantiles and stop-loss premiums. Several numerical illustrations confirm the very high accuracy of the methodology.
\end{abstract}

Keywords: life annuities, stochastic interest rates, comonotonicity, stop-loss premiums.

${ }^{*}$ Corresponding author. E-mail address: Tom.Hoedemakers@econ.kuleuven.ac.be

${ }^{\dagger}$ Department of Applied Economics, Catholic University Leuven, Naamsestraat 69, 3000 Leuven, Belgium

${ }^{\ddagger}$ University Center of Statistics, W. de Croylaan 54, 3001 Heverlee, Belgium

${ }^{\S}$ Institute of Actuarial Science, University of Amsterdam, Roeterstraat 11, 1018 WB Amsterdam, Netherlands 


\section{Introduction}

Unlike in finance, in insurance the concept of stochastic interest rates emerged quite recently. In the traditional approach to life contingencies only decrements are assumed to be stochastic — see e.g. Bowers et al. (1986), Wolthuis and Van Hoek (1986). Such a simplification allows to treat effectively summary measures of financial contracts such as the mean, the standard deviation or the upper quantiles. For a more detailed discussion about the distributions in life insurance under deterministic interest rates, see e.g. Dhaene (1990).

In non-life insurance the use of deterministic interest rates may be justified by short terms of insurance commitments. However in the case of the life insurance and the life annuity business, durations of contracts are typically very long (often 30 or even more years). Then uncertainty about future rates of return becomes very high. Moreover the financial and investment risk unlike the mortality risk - cannot be diversified with an increase in the number of policies. Therefore in order to calculate insurance premiums or mathematical reserves, actuaries are forced to adopt very conservative assumptions. As a result the diversification effects between interest rates in different investment periods may not be taken into account (i.e. that poor investment results in some periods are usually compensated by very good in others) and the life insurance business becomes too expensive, both for the insureds who have to pay higher insurance premiums and for the shareholders who have to provide more capital than necessary. For these reasons the necessity to introduce models with stochastic interest rates have been well-understood in the actuarial world.

In the actuarial literature numerous papers have treated the random interest rates. In Boyle (1976) autoregressive models of order one are introduced to model interest rates. Bellhouse \& Panjer $(1980,1981)$ use similar models to compute moments of insurance and annuity functions. In Wilkie (1976) the force of interest is assumed to follow a Gaussian random walk. Waters (1978) computes the moments of actuarial functions when the interest rates are independent and identically Gaussian distributed. He computes also moments of portfolios of policies and approximates the limiting distribution by Pearson's curves. In Dhaene (1989) the force of interest is modelled as an $\operatorname{ARIMA}(p, d, q)$ process. He uses this model to compute the moments of the present value functions. Norberg (1990) provides an axiomatic approach to stochastic interest rates and the valuation of payment streams. Parker (1994d) compares two approaches to the randomness of interest rates: by modelling only the accumulated interest and by modelling the force of interest. Both methodologies are illustrated by calculating the mean, the standard deviation and the skewness of the annuity-immediate.

An overview of stochastic life contingencies with solvency valuation is presented in Frees (1990). In the papers of Beekman and Fuelling $(1990,1991)$ the mean and the standard deviation of continuous-time life annuities are calculated with the force of mortality modelled as an OrnsteinUhlenbeck and a Wiener process respectively. In the paper of 1993 the same authors give expressions for the mean and the standard deviation of the future life insurance payments. Norberg (1993) derives the first two moments of the present value of stochastic payment streams. The first three moments of homogeneous portfolios of life insurance and endowment policies are calculated in Parker (1994a) and (1994b) and the results are generalized to heterogeneous portfolios in Parker (1997). The same author (1994c, 1996) provides a recursive formula to 
calculate an approximate distribution function of the limiting homogeneous portfolio of term life insurance and endowment policies. In Degbicka (2003) the mean and the variance are calculated for the present value of discrete-time payment streams in life insurance.

Despite the literature on stochastic interest rates in life insurance is already quite rich, most of the problems still wait for satisfactory solutions. In almost all papers the authors restrict themselves to calculating the first two or three moments of the present value function (except Waters (1978), Parker (1994d) and (1996)). The computation of the first few moments may be seen as just a first attempt to explore the properties of a random distribution. Moreover in general the variance does not appear to be the most suitable risk measure to determine the solvency requirements for an insurance portfolio. As a two-sided risk measure it takes into account both positive and negative discrepancies which leads to over- or underestimation of the reserve in the case of a skewed distribution. It doesn't also emphasize the tail properties of the distribution. In this case it seems much more appropriate to use the Value-at-Risk (the $p$-th quantile) or even the Tail Value-at-Risk (which is essentially the same as an average of all quantiles above a predefined probability level $p$ ). Also risk measures based on stop-loss premiums (for example the Expected Shortfall) can be used in this context.

In this contribution we aim to provide some conservative estimates both for high quantiles and stop-loss premiums for an individual policy and for a whole portfolio. We focus here only on life annuities, however similar techniques may be used to get analogous estimates for more general life contingencies. In order to approximate quantiles we will use the concept of comonotonicity (see Dhaene et al. (2002a) and (2002b)) which allows to substitute the sum of strongly dependent random variables by the one-dimensional distribution which is mathematically much more tractable. More precisely we will approximate the quantiles of the present value of a life annuity (or a portfolio of life annuities) by the quantiles of a distribution being an upper bound in so-called convex order sense (which will be typically a conservative estimate for a large value of $p$ ) and the quantiles of a lower bound (which provide some additional information about the quality of the approximation). We will also consider the convex combination of the upper and the lower bound which appears as a very good approximation (see Vyncke et al. (2004) for an application in the context of Asian option pricing).

We perform our analysis separately for a single life annuity and a whole portfolio of policies. Our solution enables to solve with a great accuracy the personal finance problem: How much does one need to invest now to ensure - given a periodical (e.g. yearly) consumption pattern — that the probability of outliving his or her money is very small (e.g. less than $1 \%$ )?

The case of a portfolio of life annuity policies has been studied extensively in the literature, but only in the limiting case - for homogeneous portfolios, when the mortality risk is fully diversified. However the applicability of these results in insurance practice may be questioned: especially in the case of the life annuity business a typical portfolio does not contain enough policies to speak about full diversification. For this reason we propose to approximate the number of active policies in subsequent years using a normal power distribution (by fitting the first three moments of the corresponding binomial distributions) and to model the present value of future benefits as a scalar product of mutually independent random vectors. 
The paper is organized as follows. In Section 2 we give a summary of the model assumptions and properties for the return process and the mortality process that are needed to reach our goal. Section 3 provides theoretical background for the concept of convex order, the approximations for sums and scalar products of random variables and for stop-loss premiums. In the first part of Section 4 we apply the theory to the present value of a single life annuity policy. In the second part of this section we present the convex bounds for a homogeneous portfolio of policies. A numerical illustration is provided at the end of each part. We also illustrate the obtained results graphically. Finally Section 5 summarizes and concludes our findings.

\section{The model assumptions}

\subsection{Stochastic returns}

The normality assumption for modelling returns on investment has been questioned in the short term setting (e.g. daily returns — see Schoutens (2003)). In the long term however Gaussian models provide a satisfactory approximation since the Central Limit Theorem is applicable (some empirical evidence is provided e.g. in Cesari \& Cremonini (2003)). Therefore in this contribution we restrict ourselves to two simple Gaussian models for the return on investment process $Y(t)$. More precisely, we will focus on modelling returns by means of a Brownian motion with drift (the Black \& Scholes model) and an Ornstein-Uhlenbeck process.

In the Black \& Scholes model the returns are described by the following equation:

$$
Y(t)=\mu t+\sigma B_{t}
$$

where $B_{t}$ denotes a standard Brownian motion process. It is straightforward to derive formulas for the mean and variance functions:

$$
\begin{aligned}
\mathrm{E}[Y(t)] & =\mu t \\
\operatorname{Cov}(Y(s), Y(t)) & =\sigma^{2} \min (s, t) .
\end{aligned}
$$

We use the abbreviation B-SM for this model.

In the Ornstein-Uhlenbeck model the return process is described by

$$
Y(t)=\mu t+X(t)
$$

where $X(t)$ is the solution of the following stochastic differential equation:

$$
d X(t)=-a X(t) d t+\sigma d B_{t}
$$

where $a$ and $\sigma$ are positive constants. Then $Y(t)$ is a Gaussian process with the mean and the covariance function given by:

$$
\begin{aligned}
\mathrm{E}[Y(t)] & =\mu t \\
\operatorname{Cov}(Y(s), Y(t)) & =\frac{\sigma^{2}}{2 a}\left(e^{-a|t-s|}-e^{-a(t+s)}\right) .
\end{aligned}
$$



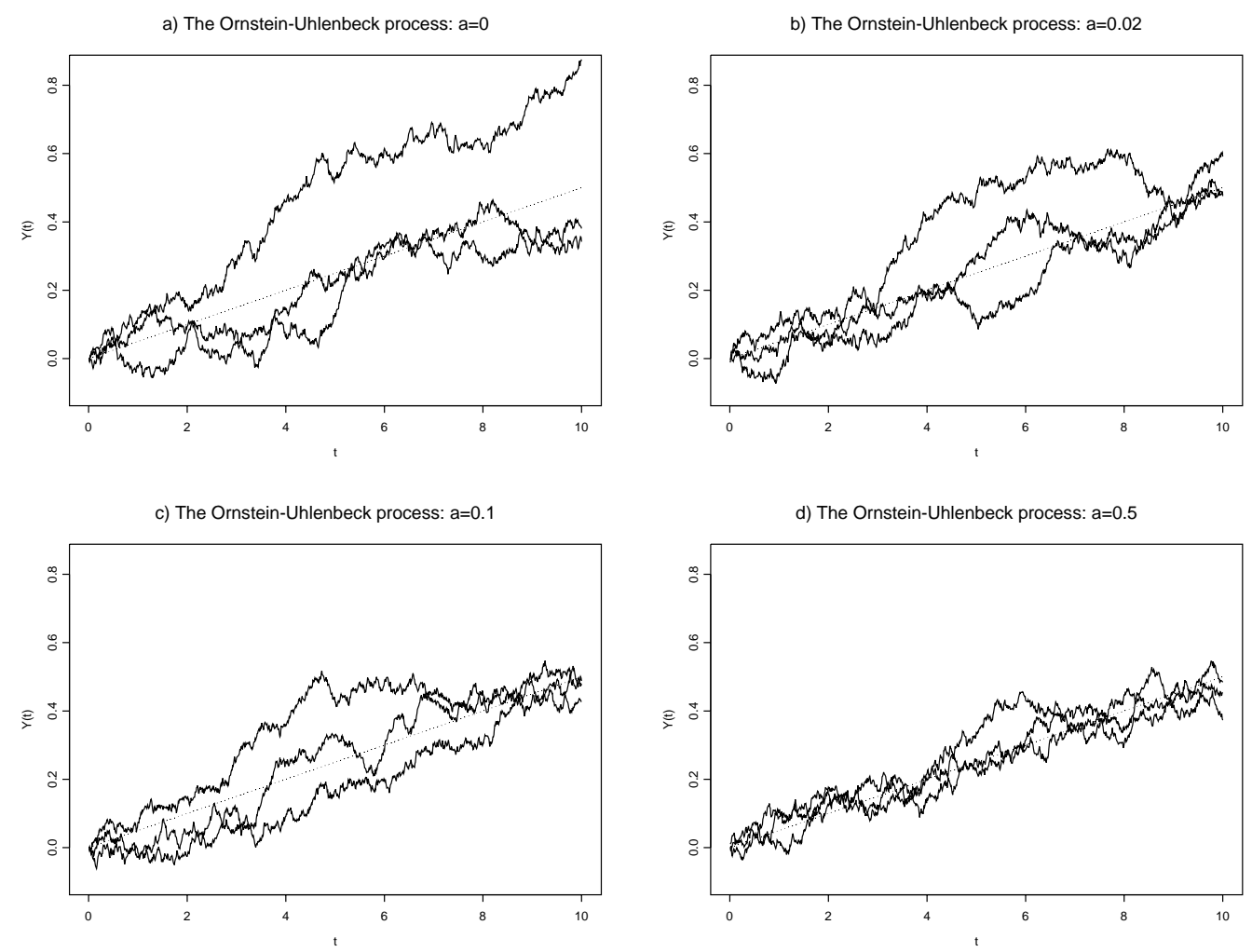

Figure 1: Typical paths for the Ornstein-Uhlenbeck process with the mean parameter $\mu=0.05$, volatility parameter $\sigma=0.07$ and different values of parameter $a$.

Note that for $a=0$ the Ornstein-Uhlenbeck process degenerates to an ordinary Brownian motion with drift and is equivalent to the Black \& Scholes setting. When $a>0$, the process $Y(t)$ has no independent increments any more. Moreover, it becomes mean reverting. Intuitively the property of mean reversion means that the process $Y(t)$ cannot deviate too far from its mean function $\mathrm{E}[Y(t)]$. In fact the parameter $a$ measures how strong the process $Y(t)$ is attracted by its mean function. The value $a=0$ means that there is no attraction and as a consequent the increments are independent. For more details we refer to Arnold (1974). The abbreviation O-UM will be used to indicate this model.

Remark that for both B-SM and O-UM models one has that $\operatorname{Cov}(Y(s), Y(t))>0$ for any $t, s>0$.

\subsection{Decrements}

A life annuity may be defined as a series of periodic payments where each payment will actually be made only if a designated life is alive at the time the payment is due. Let us consider a person aged $x$ years, also called a life aged $x$ and denoted by $(x)$. We denote his or her future lifetime by $T_{x}$. Thus $x+T_{x}$ will be the age of death of the person. The future lifetime $T_{x}$ is a 
random variable with a probability distribution function

$$
G_{x}(t)=\operatorname{Pr}\left[T_{x} \leq t\right]={ }_{t} q_{x}, \quad t \geq 0 .
$$

The function $G_{x}$ represents the probability that the person will die within $t$ years, for any fixed $t$. We assume that $G_{x}$ is known. We define $K_{x}=\left\lfloor T_{x}\right\rfloor$, the number of completed future years lived by $(x)$, or the curtate future lifetime of $(x)$, where $\lfloor$.$\rfloor is the floor function, i.e. \lfloor x\rfloor$ is the largest integer less than or equal to $x$. The probability distribution of the integer valued random variable $K_{x}$ is given by

$$
\operatorname{Pr}\left[K_{x}=k\right]=\operatorname{Pr}\left[k \leq T_{x}<k+1\right]={ }_{k+1} q_{x}-{ }_{k} q_{x}={ }_{k \mid} q_{x}, \quad k=0,1, \ldots
$$

Let us denote the total lifetime by the random variable $T$. Remark that

$$
\operatorname{Pr}\left[T_{x} \leq t\right]=\operatorname{Pr}[T \leq x+t \mid T \geq x] .
$$

With this notation, $T \equiv T_{0}$. Further, the ultimate age of the life table is denoted by $\omega$, this means that $\omega-x$ is the first remaining lifetime of $(x)$ for which ${ }_{\omega-x} q_{x}=1$, or equivalently, $G_{x}^{-1}(1)=\omega-x$.

In the remainder of this paper we will always use the standard actuarial notation:

$$
\operatorname{Pr}\left[T_{x}>t\right]={ }_{t} p_{x}, \quad \operatorname{Pr}\left[T_{x}>1\right]=p_{x}, \quad \operatorname{Pr}\left[T_{x} \leq t\right]={ }_{t} q_{x}, \quad \operatorname{Pr}\left[T_{x} \leq 1\right]=q_{x} .
$$

In this contribution we consider three types of annuities. The present value of a single life annuity for a person aged $x$ paying periodically (e.g. yearly) a fixed amount of $\alpha_{i}(i=1, \ldots,\lfloor\omega-x\rfloor)$ can be expressed as

$$
S_{s p, x}=\sum_{i=1}^{K_{x}} \alpha_{i} e^{-Y(i)}=\sum_{i=1}^{\lfloor\omega-x\rfloor} I_{\left(T_{x}>i\right)} \alpha_{i} e^{-Y(i)} .
$$

Note that $I_{(.)}$denotes the indicator function, i.e. $I_{(c)}=1$ if the condition $c$ is true and $I_{(c)}=0$ if it is not.

We consider also the present value of a homogeneous portfolio of life annuities - this random variable is particularly interesting for an insurer who has to determine a sufficient level of the reserve and the solvency margin. Assuming that every beneficiary gets a fixed amount of $\alpha_{i}$ $(i=1, \ldots,\lfloor\omega-x\rfloor)$ per year, the present value can be expressed as follows

$$
S_{p p, x}=\sum_{i=1}^{\lfloor\omega-x\rfloor} \alpha_{i} N_{i} e^{-Y(i)},
$$

where $N_{i}$ denotes the remaining number of policies-in-force in year $i$.

Finally, consider a portfolio of $N_{0}$ homogeneous life annuity contracts for which the future lifetimes of the insureds $T_{x}^{(1)}, T_{x}^{(2)}, \ldots, T_{x}^{\left(N_{0}\right)}$ are assumed to be independent. Then the insurer faces two risks: mortality risk and investment risk. Note that from the Law of Large Numbers 
the mortality risk decreases with the number of policies $N_{0}$ while the investment risk remains the same (each of the policies is exposed to the same investment risk). Thus, for sufficiently large $N_{0}$ we have that

$$
\sum_{i=1}^{\lfloor\omega-x\rfloor} \alpha_{i} N_{i} e^{-Y(i)}=N_{0}\left(\sum_{i=1}^{\lfloor\omega-x\rfloor} \alpha_{i} \frac{N_{i}}{N_{0}} e^{-Y(i)}\right) \approx N_{0}\left(\sum_{i=1}^{\lfloor\omega-x\rfloor} \alpha_{i} p_{x} e^{-Y(i)}\right) .
$$

Hence in the case of large portfolios of life annuities it suffices to compute risk measures of an 'average' portfolio $S_{a p p, x}$ given by

$$
S_{a p p, x}=\sum_{i=1}^{\lfloor\omega-x\rfloor} \alpha_{i i} p_{x} e^{-Y(i)}=\mathrm{E}\left[S_{s p, x} \mid Y(1), \cdots, Y(\lfloor\omega-x\rfloor)\right] .
$$

Obviously $S_{s p, x}, S_{p p, x}$ and $S_{a p p, x}$ depend on the distribution of the total lifetime $T$. We assume that $T$ follows the Gompertz-Makeham law, i.e. the force of mortality at age $\xi$ is given by the formula

$$
\mu_{\xi}=\alpha+\beta c^{\xi},
$$

where $\alpha>0$ is a constant component, interpreted as capturing accident hazard, and $\beta c^{\xi}$ is a variable component capturing the hazard of aging with $\beta>0$ and $c>1$. This leads to the survival probability

$$
{ }_{t} p_{x}=\operatorname{Pr}\left[T_{x}>t\right]=e^{-\int_{x}^{x+t} \mu_{\xi} d \xi}=s^{t} g^{c^{x+t}-c^{x}},
$$

where

$$
s=e^{-\alpha} \text { and } g=e^{-\frac{\beta}{\log c}} .
$$

In numerical illustrations we use the Belgian analytic life tables MR and FR for life annuity valuation, with corresponding constants $s=0.999441703848, g=0.999733441115$ and $c=$ 1.101077536030 , for males and $s=0.999669730966, g=0.999951440171$ and $c=1.116792453830$ for females.

Denote by $T^{\prime}$ and $T_{x}^{\prime}$ the corresponding random variables from the Gompertz family — the subclass of the Makeham-Gompertz family with the force of mortality given by

$$
\mu_{\xi}^{\prime}=\beta c^{\xi} .
$$

It is straightforward to show that

$$
T_{x} \stackrel{d}{=} \min \left(T_{x}^{\prime}, E / \alpha\right)
$$

where $\stackrel{d}{=}$ means equality in distribution and $E$ denotes a random variable from the standard exponential distribution, independent of $T^{\prime}$. Indeed, one has that

$$
\begin{aligned}
\operatorname{Pr}\left[\min \left(T_{x}^{\prime}, E / \alpha\right)>t\right] & =\operatorname{Pr}\left[T_{x}^{\prime}>t\right] \operatorname{Pr}[E>\alpha t] \\
& =e^{-\int_{x}^{x+t} \mu_{\xi}^{\prime} d \xi} e^{-\alpha t} \\
& =e^{-\int_{x}^{x+t} \mu_{\xi} d \xi} \\
& =\operatorname{Pr}\left[T_{x}>t\right] .
\end{aligned}
$$


The cumulative distribution function for the Gompertz law, unlike for the Makeham-Gompertz law in general, has an analytical expression for the inverse function and therefore (5) can be used for simulations.

For generating one random variate from Makeham's law, we use the composition method (Devroye, 1986) and perform the following steps

1. Generate $G$ from the Gompertz's law by the well-known inversion method

2. Generate $E$ for the exponential(1) distribution

3. Retain $T=\min (G, E / \alpha)$,

where $\alpha=-\log s$, see (4).

\section{Convex bounds for scalar products of random variables}

In many financial and actuarial applications where a sum of stochastic terms is involved, the distribution of the quantity under investigation is too difficult to obtain. It is well-known that the distribution function of a sum of dependent random variables cannot be determined analytically.

Therefore, instead of calculating the exact distribution, we will look for bounds, in the sense of "more favourable/less dangerous" and "less favourable/more dangerous", with a simpler structure. This technique is common practice in the actuarial literature. When lower and upper bounds are close to each other, together they can provide reliable information about the original and more complex variable. The notion less favourable or more dangerous variable will be defined by means of the convex order.

In a paper of 2000, Kaas, Dhaene and Goovaerts investigate the present value of a rather general cash flow, as a special case of sums of dependent risks. Making use of comonotonic risks, they derive upper and lower bounds for the distribution of the present value, in the sense of convex ordering. This means that they replace the original sum by a new sum, for which the components have the same marginal distributions as the components in the original sum, but with the most "dangerous" dependence structure. The advantage of working with a sum of comonotonic variables, has to be found in the fact that the calculation of the distribution of such a sum is quite easy. In particular this technique is very useful to find reliable estimations of upper quantiles and stop-loss premiums.

In this contribution we study sums of the form (1) and (2). Despite both equations represent sums of strongly dependent random variables, the results of Kaas et al. (2000) and Dhaene et al. (2002a) and (2002b) cannot be applied directly - the first sum has a random time horizon, in the second case the scalar product structure requires some additional comments. Similar problems have been considered in the case of loss reserving in Hoedemakers et al. (2003 and 2005) and in the context of optimal portfolio selection in Ahcan et al. (2004). 


\subsection{Convex order and comonotonicity}

In this subsection we briefly recapitulate some theoretical results of Dhaene et al. (2002a).

Definition 1 A random variable $X$ is said to precede a random variable $Y$ in the convex order sense, notation $X \leq_{c x} Y$, if any of the following conditions hold

a) $\mathrm{E}[X]=\mathrm{E}[Y]$ and $\mathrm{E}\left[(X-d)_{+}\right] \leq \mathrm{E}\left[(Y-d)_{+}\right]$for each value of $d$;

b) $\mathrm{E}[v(X)] \leq \mathrm{E}[v(Y)]$ for each convex function $v$;

c) $\mathrm{E}[X]=\mathrm{E}[Y]$ and $\mathrm{E}[u(-X)] \geq \mathrm{E}[u(-Y)]$ for each non-decreasing concave function $u$.

Therefore in a utility context, convex order represents the common preferences of all risk-averse decision makers between random variables with equal mean. It can be also proved that the same holds for the dual theory of choice under risk of Yaari (1987) - see e.g. Dhaene et al. (2002a). Thus from the viewpoint of an insurer it will be always a prudent strategy to replace a random variable $X$ by a riskier random variable $Y$. In case $X \leq_{c x} Y$, the upper tail as well as the lower tail of $Y$ are heavier than the corresponding tails of $X$, which means that extreme values are more likely to occur for $Y$ than for $X$.

As the function $v$ defined by $v(x)=x^{2}$ is a convex funtion, it follows immediately that $X \leq_{c x} Y$ implies $\operatorname{Var}[X] \leq \operatorname{Var}[Y]$. Note that the reverse implication does not hold in general, see e.g. Dhaene et al. (2002a).

Definition 2 Let $\vec{X}=\left(X_{1}, X_{2}, \ldots, X_{n}\right)$ be a random vector with marginal distributions given by $F_{X_{i}}(t)$. Then $\vec{X}$ is said to be comonotonic if there exist a random variable $Z$ and non-decreasing (non-increasing) functions $g_{1}, g_{2}, \ldots, g_{n}: \mathbb{R} \rightarrow \mathbb{R}$ such that

$$
\vec{X} \stackrel{d}{=}\left(g_{1}(Z), g_{2}(Z), \ldots, g_{n}(Z)\right) .
$$

In the previous definition, the notation $F_{X_{i}}$ is used for the distribution function of $X_{i}$, or $(t \in \mathbb{R})$

$$
F_{X_{i}}(t)=\operatorname{Pr}\left[X_{i} \leq t\right]
$$

The classical definition of the inverse of a distribution function is the non-decreasing and leftcontinuous function $F_{X_{i}}^{-1}(p)$ defined by

$$
F_{X_{i}}^{-1}(p)=\inf \left\{x \in \mathbb{R} \mid F_{X_{i}}(x) \geq p\right\}, \quad p \in[0,1],
$$

with $\inf \emptyset=+\infty$ by convention. We define $F_{X_{i}}^{-1+}(p)$ as the non-decreasing and right-continuous function

$$
F_{X_{i}}^{-1+}(p)=\sup \left\{x \in \mathbb{R} \mid F_{X_{i}}(x) \leq p\right\}, \quad p \in[0,1],
$$

with $\sup \emptyset=-\infty$ by convention.

If a random variable $S$ is expressed as a sum of random variables $\left(X_{1}, \ldots, X_{n}\right)$, replacing the copula of $\left(X_{1}, \ldots, X_{n}\right)$ by the comonotonic copula yields an upper bound for $S$ in the convex order. On the other hand, applying Jensens inequality to $S$ provides us a lower bound. This is formalized in the following theorem, which is taken from Dhaene et al. (2002a) and Kaas et al. (2000). 
Theorem 1 Consider a sum of random variables $S=X_{1}+X_{2}+\ldots+X_{n}$ and define the following related random variables:

$$
\begin{aligned}
S^{c} & =F_{X_{1}}^{-1}(U)+F_{X_{2}}^{-1}(U)+\ldots+F_{X_{n}}^{-1}(U), \\
S^{l} & =\mathrm{E}\left[X_{1} \mid \Lambda\right]+\mathrm{E}\left[X_{2} \mid \Lambda\right]+\ldots+\mathrm{E}\left[X_{n} \mid \Lambda\right],
\end{aligned}
$$

with $U$ a standard uniform random variable and $\Lambda$ an arbitrary random variable. The following relations then hold:

$$
S^{l} \leq_{c x} S \leq_{c x} S^{c}
$$

Proof. See e.g. Dhaene et al. (2002a).

The comonotonic upper bound changes the original copula, but keeps the marginal distributions unchanged. The comonotonic lower bound on the other hand, changes both the copula and the marginals involved. Intuitively, one can expect that an appropriate choice of the conditioning variable $\Lambda$ will lead to much better approximations compared to the upper bound.

The terms in the original variable $S$ and the corresponding terms in the upper bound $S^{c}$ are all identically distributed, i.e.

$$
X_{j} \stackrel{d}{=} F_{X_{j}}^{-1}(U)
$$

The upper bound $S^{c}$ is the most dangerous combination of random variables with the same marginal distributions as the original terms $X_{j}$ in $S$. Indeed, $S^{c}$ is expressed as a sum of comonotonic variables all depending on the same random variable $U$, and thus cannot be used as hedges against each other. If one can find a conditioning random variable $\Lambda$ with the property that all random variables $\mathrm{E}\left[X_{j} \mid \Lambda\right]$ are non-increasing functions of $\Lambda$ (or all are non-decreasing functions of $\Lambda$ ), then the lower bound $S^{l}=\sum_{j=1}^{n} \mathrm{E}\left[X_{j} \mid \Lambda\right]$ is a sum of $n$ comonotonic random variables.

Example 1 For the random variables $S_{a p p, x}$ and $S_{s p, x}$, as defined in Section 2.2, one has that

$$
S_{a p p, x} \leq_{c x} S_{s p, x} \quad \text { and consequently } \operatorname{Var}\left[S_{a p p, x}\right] \leq \operatorname{Var}\left[S_{s p, x}\right] .
$$

Indeed, let $\Gamma$ denote a random variable independent of $T_{x}$. Then, it follows immediately from Theorem 1 that

$$
S_{s p, x}=\sum_{i=1}^{\lfloor\omega-x\rfloor} I_{\left(T_{x}>i\right)} \alpha_{i} e^{-Y(i)} \geq_{c x} \sum_{i=1}^{\lfloor\omega-x\rfloor} \mathrm{E}\left[I_{\left(T_{x}>i\right)} \mid \Gamma\right] \alpha_{i} e^{-Y(i)}=\sum_{i=1}^{\lfloor\omega-x\rfloor}{ }_{i} p_{x} \alpha_{i} e^{-Y(i)}=S_{a p p, x} .
$$

\subsection{The generalization to scalar products}

Consider now sums of the form:

$$
S=X_{1} Y_{1}+X_{2} Y_{2}+\ldots+X_{n} Y_{n}
$$


where the random vectors $\vec{X}=\left(X_{1}, X_{2}, \ldots, X_{n}\right)$ and $\vec{Y}=\left(Y_{1}, Y_{2}, \ldots, Y_{n}\right)$ are assumed to be mutually independent. Theoretically, the techniques developed in Section 3.1 can be applied also in this case (one can take $V_{j}=X_{j} Y_{j}$ ). Such an approach is however not very practical. First of all, it is not always easy to find the marginal distributions of $V_{j}$. Secondly, it is usually very difficult to find a suitable conditioning random variable $\Lambda$, which will be a good approximation to the whole scalar product, taking into account the riskiness of the random vector $\vec{X}$ and $\vec{Y}$ simultaneously.

The following theorem provides a much more suitable approach to deal with scalar products. Before we prove the theorem we recall a helpful lemma.

Lemma 1 Assume that $\vec{X}=\left(X_{1}, X_{2}, \ldots, X_{n}\right), \vec{Y}=\left(Y_{1}, Y_{2}, \ldots, Y_{n}\right)$ and $\vec{Z}=\left(Z_{1}, Z_{2}, \ldots, Z_{n}\right)$ are non-negative random vectors and that $\vec{X}$ is mutually independent of the vectors $\vec{Y}$ and $\vec{Z}$. If for all possible outcomes $x_{1}, x_{2}, \ldots, x_{n}$ of $\vec{X}$

$$
\sum_{i=1}^{n} x_{i} Y_{i} \leq_{c x} \sum_{i=1}^{n} x_{i} Z_{i}
$$

then the corresponding scalar products are ordered in the convex order sense, i.e.

$$
\sum_{i=1}^{n} X_{i} Y_{i} \leq_{c x} \sum_{i=1}^{n} X_{i} Z_{i} .
$$

Proof. See Hoedemakers et al. (2003).

Theorem 2 Consider the following sum of random variables

$$
S=\sum_{i=1}^{n} X_{i} Y_{i} .
$$

Assume that the vectors $\vec{X}=\left(X_{1}, X_{2}, \ldots, X_{n}\right)$ and $\vec{Y}=\left(Y_{1}, Y_{2}, \ldots, Y_{n}\right)$ are mutually independent. Define the following quantities:

$$
\begin{aligned}
S^{c} & =\sum_{i=1}^{n} F_{X_{i}}^{-1}(U) F_{Y_{i}}^{-1}(V), \\
S^{l} & =\sum_{i=1}^{n} \mathrm{E}\left[X_{i} \mid \Gamma\right] \mathrm{E}\left[Y_{i} \mid \Lambda\right],
\end{aligned}
$$

where $U$ and $V$ are independent standard uniform random variables, $\Gamma$ is a random variable independent of $\vec{Y}$ and $\Lambda$ and the second conditioning random variable $\Lambda$ is independent of $\vec{X}$ and $\Gamma$. Then, the following relation holds:

$$
S^{l} \leq_{c x} S \leq_{c x} S^{c}
$$


Proof. The proof is based on a multiple application of Lemma 1.

1. First, we prove that $\sum_{i=1}^{n} X_{i} Y_{i} \leq_{c x} \sum_{i=1}^{n} F_{X_{i}}^{-1}(U) F_{Y_{i}}^{-1}(V)$.

From Theorem 1 it follows that for all possible outcomes $\left(x_{1}, x_{2}, \ldots, x_{n}\right)$ of $\vec{X}$ the following inequality holds:

$$
\sum_{i=1}^{n} x_{i} Y_{i} \leq_{c x} \sum_{i=1}^{n} F_{x_{i} Y_{i}}^{-1}(V)=\sum_{i=1}^{n} x_{i} F_{Y_{i}}^{-1}(V) .
$$

Thus from Lemma 1 it follows immediately that $\sum_{i=1}^{n} X_{i} Y_{i} \leq_{c x} \sum_{i=1}^{n} X_{i} F_{Y_{i}}^{-1}(V)$. The same reasoning can be applied to show that

$$
\sum_{i=1}^{n} X_{i} F_{Y_{i}}^{-1}(V) \leq_{c x} \sum_{i=1}^{n} F_{X_{i}}^{-1}(U) F_{Y_{i}}^{-1}(V) .
$$

2. In a similar way, one can show that

$$
\sum_{i=1}^{n} \mathrm{E}\left[X_{i} \mid \Gamma\right] \mathrm{E}\left[Y_{i} \mid \Lambda\right] \leq_{c x} \sum_{i=1}^{n} X_{i} \mathrm{E}\left[Y_{i} \mid \Lambda\right] \leq_{c x} \sum_{i=1}^{n} X_{i} Y_{i}
$$

Notice that

$$
\sum_{i=1}^{n} F_{X_{i}}^{-1}(U) F_{Y_{i}}^{-1}(V) \leq_{c x} \sum_{i=1}^{n} F_{X_{i} Y_{i}}^{-1}(U) .
$$

\subsection{Moments based approximations for distributions of scalar products}

The concept of moments based approximations of sums of random variables has already been applied in the case of the pricing of European-style Asian options (see Vyncke et al. (2004)). As approximation one choses a convex combination of the lower and the upper bound which gives a new distribution with the same first two moments as the original distribution.

More precisely, suppose that one has a scalar product $S$, its convex lower bound $S^{l}$ and its upper bound $S^{c}$ derived as described in Section 3.2. Then the corresponding variances are also ordered, i.e.

$$
\operatorname{Var}\left[S^{l}\right] \leq \operatorname{Var}[S] \leq \operatorname{Var}\left[S^{c}\right]
$$

One can prove that a random variable $S^{m}$ given by

$$
F_{S^{m}}(t)=z F_{S^{l}}(t)+(1-z) F_{S^{c}}(t)
$$

where

$$
z=\frac{\operatorname{Var}\left[S^{c}\right]-\operatorname{Var}[S]}{\operatorname{Var}\left[S^{c}\right]-\operatorname{Var}\left[S^{l}\right]}
$$

has the same first two moments as $S$. 


\subsection{Stop-loss premiums}

In this section we consider the problem of estimating stop-loss premiums for sums (of scalar products) of random variables $S=X_{1}+\ldots+X_{n}$ or $S=X_{1} Y_{1}+\ldots+X_{n} Y_{n}$ (we assume that the vectors $\left(X_{1}, \ldots, X_{n}\right)$ and $\left(Y_{1}, \ldots, Y_{n}\right)$ are mutually independent). In general it is not easy, if possible at all, to compute stop-loss premiums of random variables of this form analytically. One has to rely either on simulation methods or more-or-less accurate approximations. We will recall a powerful though simple approximation method of Dhaene et al. (2002a) and extend it to scalar products. The approximation is based on the following theorem.

Theorem 3 Let $\left(X_{1}, \ldots, X_{n}\right)$ denote a comonotonic vector with strictly increasing marginal distributions and let $S=X_{1}+\ldots+X_{n}$. Then the stop-loss premium of $S$ can be computed as follows:

$$
\mathrm{E}\left[(S-d)_{+}\right]=\sum_{i=1}^{n} \mathrm{E}\left[\left(X_{i}-F_{X_{i}}^{-1}\left(F_{S}(d)\right)\right)_{+}\right], \quad\left(F_{S}^{-1+}(0)<d<F_{S}^{-1}(1)\right) .
$$

Proof. See Dhaene et. al (2002a).

Thus Theorem 3 allows to compute analytically stop-loss premiums of the comonotonic upper bound (6). In many applications the conditioning random variable $\Lambda$ can be chosen in such a way that also the lower bound (7) can be expressed as a comonotonic sum. Then from Definition 1 and Theorem 1 one gets the following bounds for the stop-loss premiums:

$$
\mathrm{E}\left[\left(S^{l}-d\right)_{+}\right] \leq \mathrm{E}\left[(S-d)_{+}\right] \leq \mathrm{E}\left[\left(S^{c}-d\right)_{+}\right] .
$$

This method can easily be generalized to the case of scalar products of non-negative random variables. Indeed, consider the comonotonic sum

$$
\left.S^{c}\right|_{U=u}=\sum_{i=1}^{n} F_{X_{i}}^{-1}(u) F_{Y_{i}}^{-1}(V) .
$$

The stop-loss premium of $S^{c} \mid U=u$ can be easily found by applying Theorem 3 . Then, the stop-loss premium of $S^{c}$ can be computed by conditioning on the standard uniform random variable $U$

$$
\mathrm{E}\left[\left(S^{c}-d\right)_{+}\right]=\mathrm{E}\left[\mathrm{E}\left[\left(S^{c}-d\right)_{+} \mid U\right]\right]=\int_{0}^{1} \sum_{i=1}^{n} F_{X_{i}}^{-1}(u) \mathrm{E}\left[\left(Y_{i}-F_{Y_{i}}^{-1}\left(F_{S^{c} \mid U=u}(d)\right)\right)_{+}\right] \mathrm{d} u .
$$

In general it is more difficult to calculate stop-loss premiums for the lower bound. However it can be done similarly as in the case of the upper bound if one additionally assumes that the conditioning variables $\Gamma$ and $\Lambda$ for the lower bound can be chosen in such a way that for any fixed $\gamma \in \operatorname{supp}(\Gamma)$ all components $\mathrm{E}\left[X_{i} \mid \Gamma=\gamma\right] \mathrm{E}\left[Y_{i} \mid \Lambda=\lambda\right]$ are non-decreasing (or equivalently non-increasing) in $\lambda$. Then the vector

$$
\left(\mathrm{E}\left[X_{1} \mid \Gamma=\gamma\right] \mathrm{E}\left[Y_{1} \mid \Lambda\right], \mathrm{E}\left[X_{2} \mid \Gamma=\gamma\right] \mathrm{E}\left[Y_{2} \mid \Lambda\right], \ldots, \mathrm{E}\left[X_{n} \mid \Gamma=\gamma\right] \mathrm{E}\left[Y_{n} \mid \Lambda\right]\right)
$$


is comonotonic and Theorem 3 can be applied. Thus one gets

$$
\begin{aligned}
\mathrm{E}\left[\left(S^{l}-d\right)_{+}\right] & =\mathrm{E}\left[\mathrm{E}\left[\left(S^{l}-d\right)_{+} \mid \Gamma\right]\right] \\
& =\int_{0}^{1} \sum_{i=1}^{n} \mathrm{E}\left[X_{i} \mid \Gamma=F_{\Gamma}^{-1}(u)\right] \mathrm{E}\left[\left(\mathrm{E}\left[Y_{i} \mid \Lambda\right]-F_{\mathrm{E}\left[Y_{i} \mid \Lambda\right]}^{-1}\left(F_{S^{l} \mid \Gamma=F_{\Gamma}^{-1}(u)}(d)\right)\right)_{+}\right] \mathrm{d} u .
\end{aligned}
$$

Hence if only one can compute stop-loss premiums of $Y_{i}$ and $\mathrm{E}\left[Y_{i} \mid \Lambda\right]$ - one can also compute stop-loss premiums of $S^{l}$ and $S^{c}$.

Note that stop-loss premiums of $S^{m}$ can be easily calculated as

$$
\mathrm{E}\left[\left(S^{m}-d\right)_{+}\right]=z \mathrm{E}\left[\left(S^{l}-d\right)_{+}\right]+(1-z) \mathrm{E}\left[\left(S^{c}-d\right)_{+}\right] .
$$

\subsection{Discounting with Gaussian returns}

In this section we consider discounted sums of random variables as a special type of scalar products, namely

$$
S=\sum_{i=1}^{n} \alpha_{i} X_{i} e^{-Y(i)}
$$

where the vector $\vec{Y}=(Y(1), Y(2), \ldots, Y(n))$ is assumed to have a $n$-dimensional normal distribution with given mean vector

$$
\vec{\mu}=\left(\mu_{1}, \ldots, \mu_{n}\right)=(\mathrm{E}[Y(1)], \mathrm{E}[Y(2)], \ldots, \mathrm{E}[Y(n)])
$$

and covariance matrix

$$
\boldsymbol{\Sigma}=\left[\sigma_{i j}\right]_{1 \leq i, j \leq n}=[\operatorname{Cov}(Y(i), Y(j))]_{1 \leq i, j \leq n}
$$

( $\sigma_{i i}$ will be denoted by $\left.\sigma_{i}^{2}\right), \vec{X}=\left(X_{1}, X_{2}, \ldots, X_{n}\right)$ is a vector of non-negative random variables and the vectors $\vec{X}$ and $\vec{Y}$ are mutually independent. In particular we will concentrate on the case when $Y(i)$ is defined by one of the models presented in Section 2.1. Then the sum $S$ has a clear interpretation: it is the discounted value of future benefits $\alpha_{i} X_{i}$ with interest rates described by one of well-known Gaussian models. The input variables of the two discussed return models are displayed in Table 1.

We will consider the problem in general, without imposing any conditions on the random variables $X_{i}$. The specific calculations for the case of life annuities are performed in the next section.

\subsubsection{The upper bound}

From Theorem 2 it follows that

$$
\begin{aligned}
S^{c} & =\sum_{i=1}^{n} F_{X_{i}}^{-1}(U) F_{\alpha_{i} e^{-Y(i)}}^{-1}(V) \\
& =\sum_{i=1}^{n} F_{X_{i}}^{-1}(U) \alpha_{i} e^{-\mu_{i}+\operatorname{sign}\left(\alpha_{i}\right) \sigma_{i} \Phi^{-1}(V)},
\end{aligned}
$$




\begin{tabular}{l|c|l}
\hline Model & Variable & Formula \\
\hline B-SM & $\mathrm{E}[Y(i)]=\mu_{i}$ & $i \mu$ \\
& $\operatorname{Var}[Y(i)]=\sigma_{i}^{2}$ & $i \sigma^{2}$ \\
& $\operatorname{Var}[\Lambda]=\sigma_{\Lambda}^{2}$ & $\sum_{j=1}^{n} j \beta_{j}^{2} \sigma^{2}+\sum_{1 \leq j<k \leq n} 2 j \beta_{j} \beta_{k} \sigma^{2}$ \\
& $\operatorname{Cov}[Y(i), \Lambda]$ & $\sum_{j=1}^{n} \min (i, j) \beta_{j} \sigma^{2}$ \\
\hline O-UM & $\mathrm{E}[Y(i)]=\mu_{i}$ & $i \mu$ \\
& $\operatorname{Var}[Y(i)]=\sigma_{i}^{2}$ & $\frac{\sigma^{2}}{2 \alpha}\left(1-e^{-2 i \alpha}\right)$ \\
& $\operatorname{Var}[\Lambda]=\sigma_{\Lambda}^{2}$ & $\frac{\sigma^{2}}{2 \alpha}\left(\sum_{j=1}^{n} \beta_{j}^{2}\left(1-e^{-2 j \alpha}\right)+\right.$ \\
& & \multicolumn{2}{c}{$\left.+\sum_{1 \leq j<k \leq n} 2 \beta_{j} \beta_{k}\left(e^{-(k-j) \alpha}-e^{-(j+k) \alpha}\right)\right)$} \\
& & \multicolumn{2}{|c}{} \\
& $\operatorname{Cov}[Y(i), \Lambda]$ & $\frac{\sigma^{2}}{2 \alpha} \sum_{j=1}^{n} \beta_{j}\left(e^{-|i-j| \alpha}-e^{-(i+j) \alpha}\right)$ \\
\hline
\end{tabular}

Table 1: Input variables of different models for returns

where $U$ and $V$ are independent standard uniform random variables.

Obviously $\mu_{i}$ and $\sigma_{i}$ depend on the methodology used to model the returns. The cumulative distribution function of $S^{c}$ can be calculated in three steps:

1. Suppose that $U=u$ is fixed. Then from (12) it follows that conditional quantiles can be computed as

$$
F_{S^{c} \mid U=u}^{-1}(p)=\sum_{i=1}^{n} F_{X_{i}}^{-1}(u) \alpha_{i} e^{-\mu_{i}+\operatorname{sign}\left(\alpha_{i}\right) \sigma_{i} \Phi^{-1}(p)} ;
$$

2. Obviously for any $u$ the function given by (13) is continuous and strictly increasing. Thus for any $y \geq 0$ one can compute the value of the conditional distribution function using one of the well-known numerical methods (e.g. Newton-Raphson) as a solution of

$$
\sum_{i=1}^{n} F_{X_{i}}^{-1}(u) \alpha_{i} e^{-\mu_{i}+\operatorname{sign}\left(\alpha_{i}\right) \sigma_{i} \Phi^{-1}\left(F_{S^{c} \mid U=u}(y)\right)}=y
$$

3. The cumulative distribution function of $S^{c}$ can be now derived as

$$
F_{S^{c}}(y)=\int_{0}^{1} F_{S^{c} \mid U=u}(y) \mathrm{d} u
$$

Stop-loss premiums of the upper bound can be computed as follows. For simplicity of notation let us denote

$$
d_{u, i}=F_{\alpha_{i} e^{-Y(i)}}^{-1}\left(F_{S^{c} \mid U=u}(d)\right)=\alpha_{i} e^{-\mu_{i}+\operatorname{sign}\left(\alpha_{i}\right) \sigma_{i} \Phi^{-1}\left(F_{S^{c} \mid U=u}(d)\right)} .
$$

Then one has

$$
\mathrm{E}\left[\left(\alpha_{i} e^{-Y(i)}-d_{u, i}\right)_{+}\right]=\alpha_{i} e^{-\mu_{i}+\frac{\sigma_{i}^{2}}{2}} \Phi\left(\operatorname{sign}\left(\alpha_{i}\right) b_{u, i}^{(1)}\right)-d_{u, i} \Phi\left(\operatorname{sign}\left(\alpha_{i}\right) b_{u, i}^{(2)}\right),
$$


where

$$
b_{u, i}^{(1)}=\frac{-\mu_{i}+\sigma_{i}^{2}-\ln \left(d_{u, i}\right)}{\sigma_{i}}, \quad b_{u, i}^{(2)}=b_{u, i}^{(1)}-\sigma_{i}
$$

(see e.g. Dhaene et al. (2002a)). Then the stop-loss premium of $S^{c}$ with retention $d$ can be computed by plugging (16) into (10)

$$
\begin{aligned}
\mathrm{E}\left[\left(S^{c}-d\right)_{+}\right]= & \int_{0}^{1} \sum_{i=1}^{n} F_{X_{i}}^{-1}(u) \mathrm{E}\left[\left(\alpha_{i} e^{-Y(i)}-d_{u, i}\right)_{+}\right] \mathrm{d} u \\
= & \sum_{i=1}^{n} \alpha_{i} e^{-\mu_{i}+\frac{1}{2} \sigma_{i}^{2}} \int_{0}^{1} F_{X_{i}}^{-1}(u) \Phi\left(\operatorname{sign}\left(\alpha_{i}\right) \sigma_{i}-\Phi^{-1}\left(F_{S^{c} \mid U=u}(d)\right)\right) \mathrm{d} u- \\
& -d\left(1-F_{S^{c}}(d)\right) .
\end{aligned}
$$

\subsubsection{The lower bound}

The computations for the lower bound are performed similarly, however the quality of the bound

heavily depends on the choice of the conditioning random variables. Recall that from Theorem 2 it follows that

$$
S^{l}=\sum_{i=1}^{n} \mathrm{E}\left[X_{i} \mid \Gamma\right] \mathrm{E}\left[\alpha_{i} e^{-Y(i)} \mid \Lambda\right]
$$

where the first conditioning variable $\Gamma$ is independent of $\Lambda$ and $\vec{Y}$ and where the second conditioning variable $\Lambda$ is independent of $\Gamma$ and $\vec{X}$. In this section the choice of $\Gamma$ will not be discussed and the random variable $\Lambda$ will be assumed to be of the form

$$
\Lambda=\sum_{i=1}^{n} \beta_{i} Y(i)
$$

with $\beta_{i}(i=1, \ldots, n)$ a non-negative constant.

Under these assumptions the vectors of the form $(Y(i), \Lambda)$ have a bivariate normal distribution. Thus, $Y(i) \mid \Lambda=\lambda$ will be normally distributed with mean and variance given by

$$
\mu_{i, \lambda}=\mu_{i}+\frac{\operatorname{Cov}(Y(i), \Lambda)}{\operatorname{Var}[\Lambda]}(\lambda-\mathrm{E}[\Lambda])
$$

and

$$
\sigma_{i, \lambda}^{2}=\sigma_{i}^{2}-\frac{\operatorname{Cov}(Y(i), \Lambda)^{2}}{\operatorname{Var}[\Lambda]}
$$


The lower bound (18) can be written out as

$$
\begin{aligned}
S^{l} & =\sum_{i=1}^{n} \mathrm{E}\left[X_{i} \mid \Gamma\right] \mathrm{E}\left[\alpha_{i} e^{-Y(i)} \mid \Lambda\right] \\
& =\sum_{i=1}^{n} \mathrm{E}\left[X_{i} \mid \Gamma\right] \alpha_{i} e^{-\mu_{i, \Lambda}+\frac{\sigma_{i, \Lambda}^{2}}{2}} \\
& =\sum_{i=1}^{n} \mathrm{E}\left[X_{i} \mid \Gamma\right] \alpha_{i} e^{-\mu_{i}+\frac{1}{2} \sigma_{i}^{2}\left(1-r_{i}^{2}\right)-\sigma_{i} r_{i} \Phi^{-1}(U)},
\end{aligned}
$$

with $U$ a standard uniform random variable and correlations given by

$$
r_{i}=\operatorname{Corr}(Y(i), \Lambda)=\frac{\operatorname{Cov}(Y(i), \Lambda)}{\sqrt{\operatorname{Var}[Y(i)]} \sqrt{\operatorname{Var}[\Lambda]}} .
$$

Note that when the $\beta_{i}$ 's and $X_{i}$ 's are non-negative, also the $r_{i}$ 's are non-negative and the random variable $S^{l}$ is (given a value $\Gamma=\gamma$ ) the sum of the components of a comonotonic vector. Thus the cumulative distribution function of the lower bound $S^{l}$ can be computed, similar to the case of the upper bound $S^{c}$, in three steps:

1. From (20) it follows that the conditional quantiles (given $\Gamma=\gamma$ ) can be computed as

$$
F_{S^{l} \mid \Gamma=\gamma}^{-1}(p)=\sum_{i=1}^{n} \mathrm{E}\left[X_{i} \mid \Gamma=\gamma\right] \alpha_{i} e^{-\mu_{i}+\frac{1}{2} \sigma_{i}^{2}\left(1-r_{i}^{2}\right)+\sigma_{i} r_{i} \Phi^{-1}(p)}
$$

2. The conditional distribution function is computed as the solution of

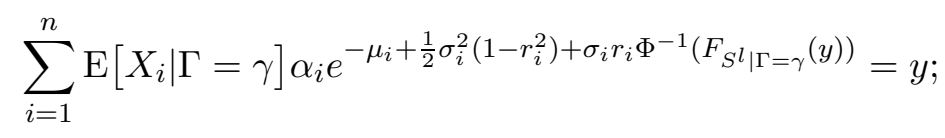

3. Finally, the cumulative distribution function of $S^{l}$ can be derived as

$$
F_{S^{l}}(y)=\int_{0}^{1} F_{S^{l} \mid \Gamma=F_{\Gamma}^{-1}(u)}(y) \mathrm{d} u .
$$

The stop-loss premiums are computed as follows. Let us denote

$$
d_{\gamma, i}=F_{\mathrm{E}\left[\alpha_{i} e^{-Y(i)} \mid \Lambda\right]}^{-1}\left(F_{S^{l} \mid \Gamma=\gamma}(d)\right)=\alpha_{i} e^{-\mu_{i}+\frac{1}{2} \sigma_{i}^{2}\left(1-r_{i}^{2}\right)+\sigma_{i} r_{i} \Phi^{-1}\left(F_{S^{l} \mid \Gamma=\gamma}(d)\right)} .
$$

Then one has

$$
\mathrm{E}\left[\left(\mathrm{E}\left[\alpha_{i} e^{-Y(i)} \mid \Lambda\right]-d_{\gamma, i}\right)_{+}\right]=\alpha_{i} e^{-\mu_{i}+\frac{1}{2} \sigma_{i}^{2}} \Phi\left(\operatorname{sign}\left(\alpha_{i}\right) b_{\gamma, i}^{(1)}\right)-d_{\gamma, i} \Phi\left(\operatorname{sign}\left(\alpha_{i}\right) b_{\gamma, i}^{(2)}\right)
$$

with

$$
b_{\gamma, i}^{(1)}=\frac{-\mu_{i}+\frac{1}{2} \sigma_{i}^{2}\left(1-r_{i}^{2}\right)+\sigma_{i}^{2} r_{i}^{2}-\ln \left(d_{\gamma, i}\right)}{\sigma_{i} r_{i}}, \quad b_{\gamma, i}^{(2)}=b_{\gamma, i}^{(1)}-\sigma_{i} r_{i}
$$


Then the stop loss-premium of $S^{l}$ with retention $d$ can be computed by plugging (23) into (11)

$$
\begin{aligned}
\mathrm{E}\left[\left(S^{l}-d\right)_{+}\right]= & \int_{0}^{1} \sum_{i=1}^{n} \mathrm{E}\left[X_{i} \mid \Gamma=F_{\Gamma}^{-1}(u)\right] \mathrm{E}\left[\left(\mathrm{E}\left[\alpha_{i} e^{-Y(i)} \mid \Lambda\right]-d_{\gamma, i}\right)_{+}\right] \mathrm{d} u \\
= & \sum_{i=1}^{n} \alpha_{i} e^{-\mu_{i}+\frac{1}{2} \sigma_{i}^{2}} \int_{0}^{1} \mathrm{E}\left[X_{i} \mid \Gamma=F_{\Gamma}^{-1}(u)\right] \Phi\left(r_{i} \sigma_{i}-\Phi^{-1}\left(F_{S^{l} \mid \Gamma=\gamma}(d)\right)\right) \mathrm{d} u- \\
& -d\left(1-F_{S^{l}}(d)\right)
\end{aligned}
$$

\subsubsection{The moments based approximations}

For the moments based approximation, as defined in (8), one has to calculate the variance of $S, S^{l}$ and $S^{c}$. In general the problem is easy solvable for the upper and the lower bound. For the exact distribution it is more difficult to find a universal solution, however it can be easily computed in the case of the single life annuity (1) and a homogeneous portfolio of life annuities (2). We will return to this problem in the next section.

Note that the upper and the lower bound of $S$, as described in subsections 3.5.1 and 3.5.2, can be seen as a special case of the following random variable $X$ with general form given by

$$
X=\sum_{i=1}^{n} \alpha_{i} f_{i}(U) g_{i}(V),
$$

where $\left(\alpha_{1}, \alpha_{2}, \ldots, \alpha_{n}\right)$ is a vector of non-negative numbers, $f_{i}$ and $g_{i}$ are non-negative functions and $U$ and $V$ two independent standard uniform random variables. Indeed, in the case of the upper bound one takes

$$
f_{i}(U)=F_{X_{i}}^{-1}(U) \quad \text { and } \quad g_{i}(V)=F_{e^{-Y(i)}}^{-1}(V)
$$

and in the case of the lower bound

$$
f_{i}(U)=\mathrm{E}\left[X_{i} \mid \Gamma\right] \text { and } g_{i}(V)=\mathrm{E}\left[e^{-Y(i)} \mid \Lambda\right] .
$$

The variance of $X$ in expression (25) can be computed as follows

$$
\begin{aligned}
\operatorname{Var}[X] & =\mathrm{E}[\operatorname{Var}[X \mid U]]+\operatorname{Var}[\mathrm{E}[X \mid U]] \\
& =\int_{0}^{1} \operatorname{Var}\left[\sum_{i=1}^{n} \alpha_{i} f_{i}(u) g_{i}(V)\right] \mathrm{d} u \\
& +\int_{0}^{1}\left(\mathrm{E}\left[\sum_{i=1}^{n} \alpha_{i} f_{i}(u) g_{i}(V)\right]\right)^{2} \mathrm{~d} u-\left(\int_{0}^{1} \mathrm{E}\left[\sum_{i=1}^{n} \alpha_{i} f_{i}(u) g_{i}(V)\right] \mathrm{d} u\right)^{2} .
\end{aligned}
$$

Thus the problem of computing the variance of $X$ is always solvable if one is able to compute the expectation and the variance of random variables $\tilde{X}$ of the form

$$
\tilde{X}=\sum_{i=1}^{n} \tilde{\alpha}_{i} g_{i}(V),
$$


for any vector of non-negative numbers $\left(\tilde{\alpha}_{1}, \tilde{\alpha}_{2}, \ldots, \tilde{\alpha}_{n}\right)$ (here $\left.\tilde{\alpha}_{i}=\alpha_{i} f_{i}(u)\right)$. For the comonotonic upper bound (12), i.e. $g_{i}(V)=e^{-\mu_{i}+\sigma_{i} \Phi^{-1}(V)}$, the variance of $\tilde{X}$ is given by

$$
\operatorname{Var}[\tilde{X}]=\sum_{i=1}^{n} \sum_{j=1}^{n} \tilde{\alpha}_{i} \tilde{\alpha}_{j} e^{-\mu_{i}-\mu_{j}+\frac{\sigma_{i}^{2}+\sigma_{j}^{2}}{2}}\left(e^{\sigma_{i} \sigma_{j}}-1\right)
$$

and for the lower bound (18), i.e. $g_{i}(V)=e^{-\mu_{i}+\frac{1}{2} \sigma_{i}^{2}\left(1-r_{i}^{2}\right)-\sigma_{i} r_{i} \Phi^{-1}(V)}$, by

$$
\operatorname{Var}[\tilde{X}]=\sum_{i=1}^{n} \sum_{j=1}^{n} \tilde{\alpha}_{i} \tilde{\alpha}_{j} e^{-\mu_{i}-\mu_{j}+\frac{\sigma_{i}^{2}+\sigma_{j}^{2}}{2}}\left(e^{r_{i} r_{j} \sigma_{i} \sigma_{j}}-1\right) .
$$

\section{The distribution of life annuities}

This section is organized into 2 subsections. In the first subsection we derive an approximation for the distribution of the present value of a single life annuity given a mortality law $T$ and a model for the returns. This distribution is very important in the context of so-called personal finance problems. Suppose that $(x)$ disposes a lump sum $L$. What is the amount that $(x)$ can yearly consume to be almost sure (i.e. sure with a sufficiently high probability e.g. $p=99 \%$ ) that the money will not be run out before death? Obviously, to answer this question one has to compute the Value-at-Risk measure of the distribution at an appropriate chosen level. In the second part of this section we will consider the distribution of a homogeneous and 'average' portfolio of life annuities. An insurer has to derive this distribution to determine the fair value of future liabilities and the solvency margin. Notice that the presented methodology is appropriate not only in the case of large portfolios when the limiting distribution can be used on the basis of the law of large numbers but also for portfolios of average size (e.g. 1000 - 5000) which are typical for the life annuity business.

\subsection{A single life annuity}

In this subsection we consider a whole life annuity of $\alpha_{i}$ payable at the end of each year $i$ while $(x)$ survives, described by the formula

$$
S_{s p, x}=\sum_{i=1}^{K_{x}} \alpha_{i} e^{-Y(i)}=\sum_{i=1}^{\lfloor\omega-x\rfloor} I_{\left(T_{x}>i\right)} \alpha_{i} e^{-Y(i)} .
$$

\subsubsection{The upper bound}

The random variable $X_{i}=I_{\left(T_{x}>i\right)}$ is $\operatorname{Bernouilli}\left({ }_{i} p_{x}\right)$ distributed and thus the inverse distribution function is given by

$$
F_{X_{i}}^{-1}(p)= \begin{cases}1 & \text { for } p>_{i} q_{x} \\ 0 & \text { for } p \leq{ }_{i} q_{x}\end{cases}
$$


This leads to the following formula for the upper bound

$$
\begin{aligned}
S_{s p, x}^{c} & =\sum_{i=1}^{\lfloor\omega-x\rfloor} F_{X_{i}}^{-1}(U) F_{\alpha_{i} e^{-Y(i)}}^{-1}(V) \\
& =\sum_{i=1}^{\left\lfloor F_{T_{x}}^{-1}(U)\right\rfloor} F_{\alpha_{i} e^{-Y(i)}}^{-1}(V),
\end{aligned}
$$

where $U$ and $V$ are independent standard uniformly distributed random variables. Thus the conditional quantiles are given by

$$
F_{S_{s p, x}^{c} \mid T_{x}=t}^{-1}(p)=\sum_{i=1}^{\lfloor t\rfloor} F_{\alpha_{i} e^{-Y(i)}}^{-1}(V)
$$

and the conditional distribution function can be computed numerically from the identity

$$
\sum_{i=1}^{\lfloor t\rfloor} \alpha_{i} e^{-\mu_{i}+\operatorname{sign}\left(\alpha_{i}\right) \sigma_{i} \Phi^{-1}\left(F_{S_{s p, x}^{c} \mid T_{x}=t}(y)\right)}=\sum_{i=1}^{k} \alpha_{i} e^{-\mu_{i}+\operatorname{sign}\left(\alpha_{i}\right) \sigma_{i} \Phi^{-1}\left(F_{S_{s p, x}^{c} \mid K_{x}=k}(y)\right)}=y .
$$

Let us denote $S_{s p, x} \mid K_{x}=k$ by $\tilde{S}_{k}$ defined as follows

$$
\tilde{S}_{k}=\sum_{i=1}^{k} \alpha_{i} e^{-Y(i)}
$$

Hence, the distribution function of $S_{s p, x}^{c}$ can be computed as

$$
\begin{aligned}
F_{S_{s p, x}^{c}}(y) & =\sum_{k=1}^{\lfloor\omega-x\rfloor} \operatorname{Pr}\left[K_{x}=k\right] F_{S_{s p, x}^{c} \mid K_{x}=k}(y) \\
& =\sum_{k=1}^{\lfloor\omega-x\rfloor} k \mid q_{x} F_{\tilde{S}_{k}^{c}}(y) \\
& =\sum_{k=1}^{\lfloor\omega-x\rfloor} k_{x} \operatorname{Pr}\left[\sum_{i=1}^{k} \alpha_{i} e^{-\mu_{i}+\operatorname{sign}\left(\alpha_{i}\right) \sigma_{i} \Phi^{-1}(U)} \leq y\right],
\end{aligned}
$$

with $\tilde{S}_{k}^{c}=\sum_{i=1}^{k} F_{\alpha_{i} e^{-Y(i)}}^{-1}(U)$ and $U$ a standard uniform random variable.

The computation of the corresponding stop-loss premiums is also straightforward:

$$
\begin{aligned}
\mathrm{E}\left[\left(S_{s p, x}^{c}-d\right)_{+}\right] & =\mathrm{E}_{K_{x}}\left[\mathrm{E}\left[\left(S_{s p, x}^{c}-d\right)_{+} \mid K_{x}\right]\right] \\
& =\sum_{k=1}^{\lfloor\omega-x\rfloor} k \mid q_{x} \mathrm{E}\left[\left(\tilde{S}_{k}^{c}-d\right)_{+}\right] \\
& =\sum_{k=1}^{\lfloor\omega-x\rfloor} k \mid q_{x}\left(\sum_{i=1}^{k} \mathrm{E}\left[\left(\alpha_{i} e^{-Y(i)}-d_{k, i}^{c}\right)_{+}\right]\right)
\end{aligned}
$$


where $d_{k, i}^{c}$ is defined analogously to (15) as

$$
d_{k, i}^{c}=\alpha_{i} e^{-\mu_{i}+\operatorname{sign}\left(\alpha_{i}\right) \sigma_{i} \Phi^{-1}\left(F_{\tilde{S}_{k}^{c}}(d)\right)}
$$

and the values of $\mathrm{E}\left[\left(\alpha_{i} e^{-Y(i)}-d_{k, i}\right)_{+}\right]$are computed like in (16). The stop-loss premium of $S_{s p, x}^{c}$ at retention $d$ can be written out explicitly as follows

$$
\mathrm{E}\left[\left(S_{s p, x}^{c}-d\right)_{+}\right]=\sum_{k=1}^{\lfloor\omega-x\rfloor}{ }_{k \mid} q_{x}\left\{\sum_{i=1}^{k} \alpha_{i} e^{-\mu_{i}+\frac{\sigma_{i}^{2}}{2}} \Phi\left(\operatorname{sign}\left(\alpha_{i}\right) \sigma_{i}-\Phi^{-1}\left(F_{\tilde{S}_{k}^{c}}(d)\right)\right)-d\left(1-F_{\tilde{S}_{k}^{c}}(d)\right)\right\} .
$$

\subsubsection{The lower bound}

For the lower bound one faces the problem of choosing appropriate conditioning random variables $\Gamma$ and $\Lambda$. The random variables $X_{i}$ are in fact comonotonic and depend only on the future lifetime $T_{x}$, thus $\Gamma=T_{x}$ is the most natural choice. As a result one simply gets

$$
\mathrm{E}\left[I_{\left(T_{x}>i\right)} \mid T_{x}\right]=I_{\left(T_{x}>i\right)}
$$

The choice of the second conditioning random variable $\Lambda$ of the form (19) is less obvious. We propose two different approaches based on Vanduffel, Hoedemakers and Dhaene (2004):

1. $\Lambda^{(a)}=\sum_{i=1}^{\lfloor\omega-x\rfloor}{ }_{i} p_{x} \alpha_{i} e^{-\mu_{i}+\frac{1}{2} \sigma_{i}^{2}} Y(i)$. Intuitively it means that the conditioning random variable is chosen as a first order approximation to the present value of the limiting portfolio $S_{a p p, x}$ in (3).

2. Consider the 'maximal variance' conditioning random variables of the form $\Lambda_{j}=\sum_{i=1}^{j} \alpha_{i} e^{-\mu_{i}+\frac{1}{2} \sigma_{i}^{2}} Y(i)(j=1, \ldots,\lfloor\omega-x\rfloor)$ and the corresponding lower bounds

$$
S_{s p, x}^{l, j}=\sum_{i=1}^{K_{x}} \mathrm{E}\left[\alpha_{i} e^{-Y(i)} \mid \Lambda_{j}\right], \quad j=1, \ldots,\lfloor\omega-x\rfloor
$$

from which one chooses the lower bound with the largest variance. The corresponding conditioning random variable will be denoted as $\Lambda^{(m)}$. This choice can be motivated as follows. For two random variables $X$ and $Y$ with $X \leq_{c x} Y$ one has that $\operatorname{Var}[X] \leq \operatorname{Var}[Y]$. Hence one can expect that a lower bound with a larger variance will provide a better fit to the original random variable.

Having chosen the conditioning random variable $\Lambda$ one proceeds as in the case of the upper bound: the first step requires the computation of the conditional distribution of the lower bound from the formula

$$
\sum_{i=1}^{k} \alpha_{i} e^{-\mu_{i}+\frac{1}{2} \sigma_{i}^{2}\left(1-r_{i}^{2}\right)+\sigma_{i} r_{i} \Phi^{-1}\left(F_{S_{s p, x} \mid K_{x}=k}(y)\right)}=y
$$


The cumulative distribution function of $S_{s p, x}^{l}$ can then be computed as

$$
\begin{aligned}
F_{S_{s p, x}^{l}}(y) & =\sum_{k=1}^{\lfloor\omega-x\rfloor} k \mid q_{x} F_{S_{s p, x}^{l} \mid K_{x}=k}(y) \\
& =\sum_{k=1}^{\lfloor\omega-x\rfloor} k \mid q_{x} F_{\tilde{S}_{k}^{l}}(y) \\
& =\sum_{k=1}^{\lfloor\omega-x\rfloor} k \mid q_{x} \operatorname{Pr}\left[\sum_{i=1}^{k} \alpha_{i} e^{-\mu_{i}-r_{i} \sigma_{i} \Phi^{-1}(U)+\frac{1}{2}\left(1-r_{i}^{2}\right) \sigma_{i}^{2}} \leq y\right],
\end{aligned}
$$

with $\tilde{S}_{k}^{l}=\mathrm{E}\left[\tilde{S}_{k} \mid \Lambda\right]$ and $U$ a standard uniform random variable.

The computation of the corresponding stop-loss premium is similar to the one of the upper bound and as a result one gets the following explicit solution

$$
\begin{aligned}
\mathrm{E}\left[\left(S_{s p, x}^{l}-d\right)_{+}\right] & =\mathrm{E}_{K_{x}}\left[\mathrm{E}\left[\left(S_{s p, x}^{l}-d\right)_{+} \mid K_{x}\right]\right] \\
& =\sum_{k=1}^{\lfloor\omega-x\rfloor} k \mid q_{x} \mathrm{E}\left[\left(\tilde{S}_{k}^{l}-d\right)_{+}\right] \\
& =\sum_{k=1}^{\lfloor\omega-x\rfloor} k \mid q_{x}\left(\sum_{i=1}^{k} \mathrm{E}\left[\left(\mathrm{E}\left[\alpha_{i} e^{-Y(i)} \mid \Lambda\right]-d_{k, i}^{l}\right)_{+}\right]\right)
\end{aligned}
$$

with $d_{k, i}^{l}$ given by

$$
d_{k, i}^{l}=\alpha_{i} e^{-\mu_{i}+\frac{1}{2} \sigma_{i}^{2}\left(1-r_{i}^{2}\right)+\sigma_{i} r_{i} \Phi^{-1}\left(F_{S_{s p, x}^{l} \mid K_{x}=k}(d)\right)} .
$$

Note that the values of $\mathrm{E}\left[\left(\mathrm{E}\left[\alpha_{i} e^{-Y(i)} \mid \Lambda\right]-d_{k, i}^{l}\right)_{+}\right]$can be computed as in (23). The stop-loss premium of $S_{s p, x}^{l}$ at retention $d$ can be written out explicitly as follows

$$
\mathrm{E}\left[\left(S_{s p, x}^{l}-d\right)_{+}\right]=\sum_{k=1}^{\lfloor\omega-x\rfloor}{ }_{k} \mid q_{x}\left\{\sum_{i=1}^{k} \alpha_{i} e^{-\mu_{i}+\frac{\sigma_{i}^{2}}{2}} \Phi\left(r_{i} \sigma_{i}-\Phi^{-1}\left(F_{\tilde{S}_{k}^{l}}(d)\right)\right)-d\left(1-F_{\tilde{S}_{k}^{l}}(d)\right)\right\} .
$$

\subsubsection{The lower bound based on a lifetime dependent conditioning random variable}

In this subsection we show how it is possible to improve the lower bound of a scalar product if one of the vectors is comonotonic. We state this result in the following lemma.

Lemma 2 Consider a scalar product of random variables $S=\sum_{i=1}^{n} X_{i} Y_{i}$, where the random vectors $\vec{X}$ and $\vec{Y}$ are independent and $\vec{X}$ is additionally assumed to be comonotonic, i.e. $\vec{X}=$ $\left(F_{X_{1}}^{-1}(U), F_{X_{2}}^{-1}(U), \ldots, F_{X_{n}}^{-1}(U)\right)$. Let $\Lambda(u)$ be a random variable which is defined for each $u \in$ $(0,1)$ separately. Define conditional random variables $S^{c l}(u)=\left.S^{c l}\right|_{U=u}$ as

$$
S^{c l}(u)=\sum_{i=1}^{n} F_{X_{i}}^{-1}(u) \mathrm{E}\left[Y_{i} \mid \Lambda(u)\right]
$$


and define the random variable $S^{c l}$ through its distribution function

$$
F_{S^{c l}}(y)=\int_{0}^{1} F_{S^{c l} \mid U=u}(y) \mathrm{d} u .
$$

Then $S^{c l} \leq_{c x} S$.

Remark: Obviously the conditioning random variable $U$ can be replaced by any other random variable which determines the comonotonic vector $\vec{X}$ by a functional relationship. In this section we consider the case when $X_{i}=I_{\left(T_{x}>i\right)}=I_{\left(K_{x} \geq i\right)}$ and therefore it is convenient to condition on the future lifetime $K_{x}$.

Proof. Let $S(u)$ denote a random variable distributed as $S$ given that $U=u$. From Definition 1 b of convex order, it follows immediately that $S^{c l}(u) \leq_{c x} S(u)$.

Indeed, let $v($.$) be an arbitrary convex function. Then we get$

$$
\mathrm{E}\left[v\left(S^{c l}\right)\right]=\int_{0}^{1} \mathrm{E}\left[v\left(S^{c l}(u)\right)\right] \mathrm{d} u \leq \int_{0}^{1} \mathrm{E}[v(S(u))] \mathrm{d} u=\mathrm{E}[v(S)],
$$

what completes the proof.

Because of Lemma 2, one can determine a lower bound of a single life annuity using the following conditioning random variable:

$$
\Lambda_{K_{x}}=\sum_{i=1}^{K_{x}} \alpha_{i} e^{-\mu_{i}+\frac{1}{2} \sigma_{i}^{2}} Y(i) .
$$

Intuitively it is clear that the lower bound defined by the random variable $\Lambda_{K_{x}}$ should approximate the underlying distribution better than those defined by the conditioning random variables $\Lambda^{(a)}$ and $\Lambda^{(m)}$. As before, one starts with computing the conditional distributions for the lower bound $S_{s p, x}^{c l}$ numerically by considering the equation

$$
\sum_{i=1}^{k} \alpha_{i} e^{-\mu_{i}+\frac{1}{2}\left(1-r_{i, k}^{2}\right) \sigma_{i}^{2}+r_{i, k} \sigma_{i} \Phi^{-1}\left(F_{S_{s p, x}^{c l} \mid K_{x}=k}(y)\right)}=y
$$

with correlations $r_{i, k}$ given by

$$
r_{i, k}=\frac{\operatorname{Cov}\left(Y(i), \Lambda_{k}\right)}{\sqrt{\operatorname{Var}[Y(i)]} \sqrt{\operatorname{Var}\left[\Lambda_{k}\right]}}
$$

Consequently, the distribution function of $S_{s p, x}^{c l}$ can be obtained as

$$
F_{S_{s p, x}^{c l}}(y)=\sum_{k=1}^{\lfloor\omega-x\rfloor} \operatorname{Pr}\left[K_{x}=k\right] F_{S_{s p, x}^{c l} \mid K_{x}=k}(y)=\sum_{k=1}^{\lfloor\omega-x\rfloor}{ }_{k \mid} q_{x} F_{\tilde{S}_{k}^{c l}}(y),
$$

with

$$
\tilde{S}_{k}^{c l}=\mathrm{E}\left[\tilde{S}_{k} \mid \Lambda_{k}\right]
$$


The stop-loss premiums of $S_{s p, x}^{c l}$ can be computed as follows

$$
\begin{aligned}
\mathrm{E}\left[\left(S_{s p, x}^{c l}-d\right)_{+}\right] & =\mathrm{E}_{K_{x}}\left[\mathrm{E}\left[\left(S_{s p, x}^{c l}-d\right)_{+} \mid K_{x}\right]\right] \\
& =\sum_{k=1}^{\lfloor\omega-x\rfloor}{ }_{k} q_{x} \mathrm{E}\left[\left(\tilde{S}_{k}^{c l}-d\right)_{+}\right] \\
& =\sum_{k=1}^{\lfloor\omega-x\rfloor} k \mid q_{x}\left(\sum_{i=1}^{k} \mathrm{E}\left[\left(\mathrm{E}\left[\alpha_{i} e^{-Y(i)} \mid \Lambda_{k}\right]-d_{k, i}^{c l}\right)_{+}\right]\right)
\end{aligned}
$$

with $d_{k, i}^{c l}$ given by

$$
d_{k, i}^{c l}=\alpha_{i} e^{-\mu_{i}+\frac{1}{2} \sigma_{i}^{2}\left(1-r_{i, k}^{2}\right)+\sigma_{i} r_{i, k} \Phi^{-1}\left(F_{\tilde{S}_{k}^{c l}}(d)\right)} .
$$

The stop-loss premium of $S_{s p, x}^{c l}$ at retention $d$ can be written out explicitly as follows

$$
\mathrm{E}\left[\left(S_{s p, x}^{c l}-d\right)_{+}\right]=\sum_{k=1}^{\lfloor\omega-x\rfloor} k \mid q_{x}\left\{\sum_{i=1}^{k} \alpha_{i} e^{-\mu_{i}+\frac{\sigma_{i}^{2}}{2}} \Phi\left(r_{i, k} \sigma_{i}-\Phi^{-1}\left(F_{\tilde{S}_{k}^{c l}}(d)\right)\right)-d\left(1-F_{\tilde{S}_{k}^{c l}}(d)\right)\right\} .
$$

\subsubsection{The moments based approximation}

Having computed the upper bound $S_{s p, x}^{c}$ and the lower bounds $S_{s p, x}^{l}$ and $S_{s p, x}^{c l}$ like in Sections 4.1.1 - 4.1.3 one can compute two moments based approximations as described in Section 3.3. To find the coefficient $z$ given by (9) one needs to calculate the variances of $S_{s p, x}^{c}, S_{s p, x}^{l}, S_{s p, x}^{c l}$ and $S_{s p, x}$. The variance of $S_{s p, x}^{c}$ and $S_{s p, x}^{l}$ can be computed as explained in Section 3.5.3. The variance of $S_{s p, x}$ and $S_{s p, x}^{c l}$ can be treated very similarly. Indeed, after some simple calculations one gets

$$
\begin{aligned}
\operatorname{Var}\left[S_{s p, x}^{c l}\right] & =\mathrm{E}_{K_{x}}\left[\mathrm{E}\left[\left(S_{s p, x}^{c l}\right)^{2} \mid K_{x}\right]\right]-\left(\mathrm{E}\left[S_{s p, x}^{c l}\right]\right)^{2} \\
& =\sum_{k=1}^{\lfloor\omega-x\rfloor} k \mid q_{x} \mathrm{E}\left[\left(\tilde{S}_{k}^{c l}\right)^{2}\right]-\left(\mathrm{E}\left[S_{s p, x}^{c l}\right]\right)^{2}, \\
\operatorname{Var}\left[S_{s p, x}\right] & =\mathrm{E}_{K_{x}}\left[\mathrm{E}\left[\left(S_{s p, x}\right)^{2} \mid K_{x}\right]\right]-\left(\mathrm{E}\left[S_{s p, x}\right]\right)^{2} \\
& =\sum_{k=1}^{\lfloor\omega-x\rfloor} k \mid q_{x} \mathrm{E}\left[\left(\tilde{S}_{k}\right)^{2}\right]-\left(\mathrm{E}\left[S_{s p, x}\right]\right)^{2},
\end{aligned}
$$


where $\tilde{S}_{k}^{c l}$ and $\tilde{S}_{k}$ are defined as in (27) and (26) respectively. Thus it suffices to plug in

$$
\begin{aligned}
\mathrm{E}\left[\tilde{S}_{k}^{c l}\right]=\mathrm{E}\left[\tilde{S}_{k}\right] & =\sum_{i=1}^{k} \alpha_{i} e^{-\mu_{i}+\frac{\sigma_{i}^{2}}{2}}, \\
\mathrm{E}\left[\left(\tilde{S}_{k}^{c l}\right)^{2}\right] & =\sum_{i=1}^{k} \sum_{j=1}^{k} \alpha_{i} \alpha_{j} e^{-\mu_{i}-\mu_{j}+\frac{1}{2}\left(\sigma_{i}^{2}+\sigma_{j}^{2}\right)+r_{i, k} r_{j, k} \sigma_{i} \sigma_{j}}, \\
\mathrm{E}\left[\left(\tilde{S}_{k}\right)^{2}\right] & =\sum_{i=1}^{k} \sum_{j=1}^{k} \alpha_{i} \alpha_{j} e^{-\mu_{i}-\mu_{j}+\frac{1}{2}\left(\sigma_{i}^{2}+\sigma_{j}^{2}\right)+\sigma_{i j}},
\end{aligned}
$$

and

$$
\mathrm{E}\left[S_{s p, x}\right]=\mathrm{E}\left[S_{s p, x}^{c l}\right]=\sum_{k=1}^{\lfloor\omega-x\rfloor} k\left|q_{x} \mathrm{E}\left[\tilde{S}_{k}\right]=\sum_{k=1}^{\lfloor\omega-x\rfloor} k\right| q_{x} \mathrm{E}\left[\tilde{S}_{k}^{c l}\right] .
$$

Now one can compute distributions of the moment based approximations from the formulas

$$
\begin{aligned}
& F_{S_{s p, x}^{m}}(y)=z_{1} F_{S_{s p, x}^{l}}(y)+\left(1-z_{1}\right) F_{S_{s p, x}^{c}}(y), \\
& F_{S_{s p, x}^{c m}}(y)=z_{2} F_{S_{s p, x}^{c l}}(y)+\left(1-z_{2}\right) F_{S_{s p, x}^{c}}(y)
\end{aligned}
$$

and their corresponding stop-loss premiums as

$$
\begin{aligned}
& \mathrm{E}\left[\left(S_{s p, x}^{m}-d\right)_{+}\right]=z_{1} \mathrm{E}\left[\left(S_{s p, x}^{l}-d\right)_{+}\right]+\left(1-z_{1}\right) \mathrm{E}\left[\left(S_{s p, x}^{c}-d\right)_{+}\right], \\
& \mathrm{E}\left[\left(S_{s p, x}^{c m}-d\right)_{+}\right]=z_{2} \mathrm{E}\left[\left(S_{s p, x}^{c l}-d\right)_{+}\right]+\left(1-z_{2}\right) \mathrm{E}\left[\left(S_{s p, x}^{c}-d\right)_{+}\right],
\end{aligned}
$$

where

$$
z_{1}=\frac{\operatorname{Var}\left[S_{s p, x}^{c}\right]-\operatorname{Var}\left[S_{s p, x}\right]}{\operatorname{Var}\left[S_{s p, x}^{c}\right]-\operatorname{Var}\left[S_{s p, x}^{l}\right]} \text { and } z_{2}=\frac{\operatorname{Var}\left[S_{s p, x}^{c}\right]-\operatorname{Var}\left[S_{s p, x}\right]}{\operatorname{Var}\left[S_{s p, x}^{c}\right]-\operatorname{Var}\left[S_{s p, x}^{c l}\right]}
$$

\subsubsection{A numerical illustration}

We examine the accuracy and efficiency of the derived approximations for a single life annuity of a 65-years old male person with yearly unit payments. We restrict ourselves to the case of a Black \& Scholes setting (model BS) with drift $\mu=0.05$ and volatility $\sigma=0.1$. We assume further that the future lifetime $T_{65}$ follows the Makeham-Gompertz law with the corresponding coefficients of the Belgian analytic life table MR (see Section 2.2). We compare the distribution functions of the upper bound $S_{s p, 65}^{c}$ and the lower bounds $S_{s p, 65}^{l}$ and $S_{s p, 65}^{c l}$, as described in the previous sections, with the original distribution function of $S_{s p, 65}$ based on extensive Monte Carlo (MC) simulation. We generated $500 \times 100000$ paths and for each estimate we computed the standard error (s.e.). As is well-known, the (asymptotic) 95\% confidence interval is given by the estimate plus or minus 1.96 times the standard error. Note also that the random paths are based on antithetic variables in order to reduce the variance. Remark that to compute the lower bound we use as conditioning random variable $\Lambda^{(m)}=\Lambda_{24}$ (the value $j=24$ was found to be the one that maximizes the variance as described in Section 4.1.2). 
Figure 2 shows the cumulative distribution functions (cdf's) of the approximations, compared to the empirical distribution. One can see that the lower bound $S_{s p, 65}^{c l}$ is almost indistinguishable from the original distribution. In order to have a better view on the behavior of the approximations in the tail, we consider a QQ-plot where the quantiles of $S_{s p, 65}^{l}, S_{s p, 65}^{c l}$ and $S_{s p, 65}^{c}$ are plotted against the quantiles of $S_{s p, 65}$ obtained by simulation. The different bounds will be good approximations if the plotted points $\left(F_{S_{s p, 65}}^{-1}(p), F_{S_{s p, 65}^{l}}^{-1}(p)\right),\left(F_{S_{s p, 65}}^{-1}(p), F_{S_{s p, 65}^{c l}}^{-1}(p)\right)$ and $\left(F_{S_{s p, 65}}^{-1}(p), F_{S_{s p, 65}^{c}}^{-1}(p)\right)$ for all values of $p$ in $(0,1)$ do not deviate too much from the line $y=x$. From the QQ-plot in Figure 3, we can conclude that the comonotonic upper bound slightly overestimates the tails of $S_{s p, 65}$, whereas the accuracy of the lower bounds $S_{s p, 65}^{l}$ and $S_{s p, 65}^{c l}$ is extremely high; the corresponding QQ-plot seems to be a perfect straight line. These visual observations are confirmed by the numerical values of some upper quantiles displayed in Table 2 , which also reports the moments based approximations $S_{s p, 65}^{m}$ and $S_{s p, 65}^{c m}$.

Stop-loss premiums for the different approximations are compared in Figure 4 and Table 3. This study confirms the high accuracy of the derived bounds. Note that for very high values of $d$ the differences become larger, however these cases don't represent any practical importance. All Monte Carlo estimates are very close to $\mathrm{E}\left[\left(S_{s p, 65}^{c l}-d\right)_{+}\right]$and some of them even turn out to be smaller than this lower bound for $\mathrm{E}\left[\left(S_{s p, 65}-d\right)_{+}\right]$. This not only demonstrates the difficulty of estimating stop-loss premiums by simulation, but it also indicates the accuracy of the lower bound $\mathrm{E}\left[\left(S_{s p, 65}^{c l}-d\right)_{+}\right]$. Indeed, since the Monte Carlo estimate is based on random paths, it can be smaller than $\mathrm{E}\left[\left(S_{s p, 65}^{c l}-d\right)_{+}\right]$and this is very likely to happen if the lower bound is close to the real stop-loss premium.

\begin{tabular}{lllllll}
\hline$p$ & $S_{s p, 65}^{l}$ & $S_{s p, 65}^{c l}$ & $S_{s p, 65}^{m}$ & $S_{s p, 65}^{c m}$ & $S_{s p, 65}^{c}$ & MC $\left(\right.$ s.e. $\left.\times 10^{3}\right)$ \\
\hline 0.995 & 27.5124 & 27.6700 & 27.7498 & 27.6943 & 30.2983 & $27.6933(6.324)$ \\
0.975 & 22.2495 & 22.2875 & 22.3559 & 22.2986 & 23.6574 & $22.2839(2.816)$ \\
0.95 & 19.9565 & 19.9713 & 20.0232 & 19.9783 & 20.8754 & $19.9731(1.896)$ \\
0.90 & 17.5905 & 17.5972 & 17.6250 & 17.6008 & 18.0797 & $17.5969(1.420)$ \\
0.75 & 14.1741 & 14.1887 & 14.1750 & 14.1887 & 14.1867 & $14.1887(0.978)$ \\
\hline
\end{tabular}

Table 2: Approximations for some selected quantiles with probability level $p$ of $S_{s p, 65}$.

\begin{tabular}{lllllll}
\hline$d$ & $S_{s p, 65}^{l}$ & $S_{s p, 65}^{c l}$ & $S_{s p, 65}^{m}$ & $S_{s p, 65}^{c m}$ & $S_{s p, 65}^{c}$ & MC (s.e. $\left.\times 10^{4}\right)$ \\
\hline 0 & 11.0944 & 11.0944 & 11.0944 & 11.0944 & 11.0944 & $11.0937(9.43)$ \\
5 & 6.3715 & 6.3756 & 6.3721 & 6.3756 & 6.3792 & $6.3748(8.67)$ \\
10 & 2.5956 & 2.6071 & 2.6029 & 2.6078 & 2.6900 & $2.6068(5.89)$ \\
15 & 0.7151 & 0.7201 & 0.7265 & 0.7213 & 0.8629 & $0.7201(0.34)$ \\
20 & 0.1628 & 0.1664 & 0.1698 & 0.1671 & 0.2536 & $0.1668(0.21)$ \\
25 & 0.0357 & 0.0379 & 0.0388 & 0.0382 & 0.0758 & $0.0382(0.10)$ \\
30 & 0.0080 & 0.0091 & 0.0092 & 0.0092 & 0.0239 & $0.0093(0.02)$ \\
35 & 0.0019 & 0.0023 & 0.0024 & 0.0023 & 0.0081 & $0.0024(0.004)$ \\
\hline
\end{tabular}

Table 3: Approximations for some selected stop-loss premia with retention $d$ of $S_{s p, 65}$. 


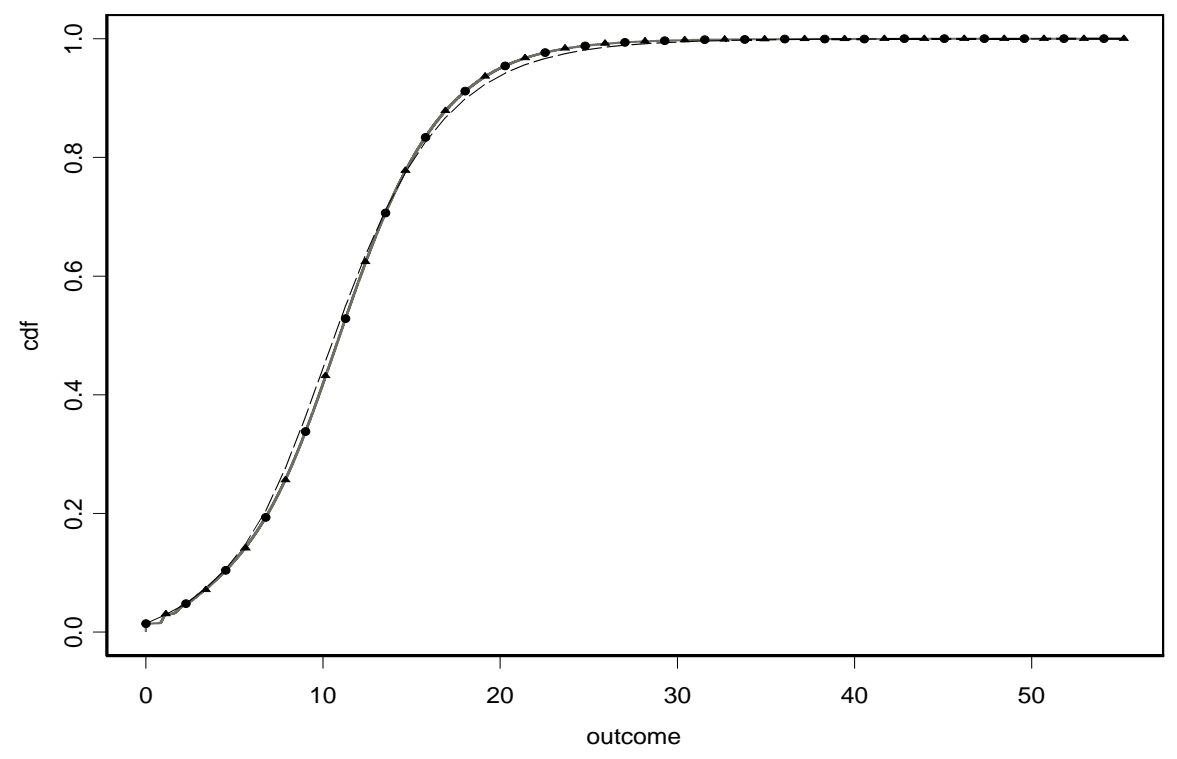

Figure 2: The cdf's of ' $S_{s p, 65}$ ' (MC) (solid grey line), $S_{s p, 65}^{l}\left(\bullet-\right.$ line), $S_{s p, 65}^{c l}$ (८-line) and $S_{s p, 65}^{c}$ (dashed line).

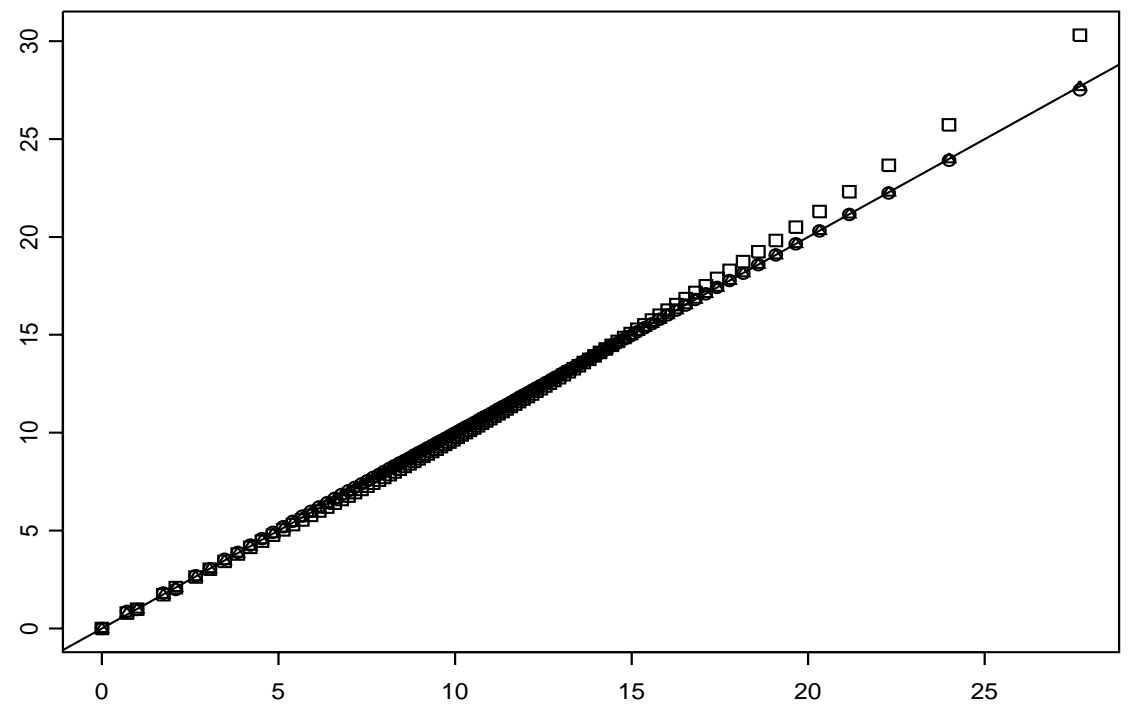

Figure 3: QQ-plot of the quantiles of $S_{s p, 65}^{l}(\circ), S_{s p, 65}^{c l}(\triangle)$ and $S_{s p, 65}^{c}(\square)$ versus those of ' $S_{s p, 65}$ ' (MC). 


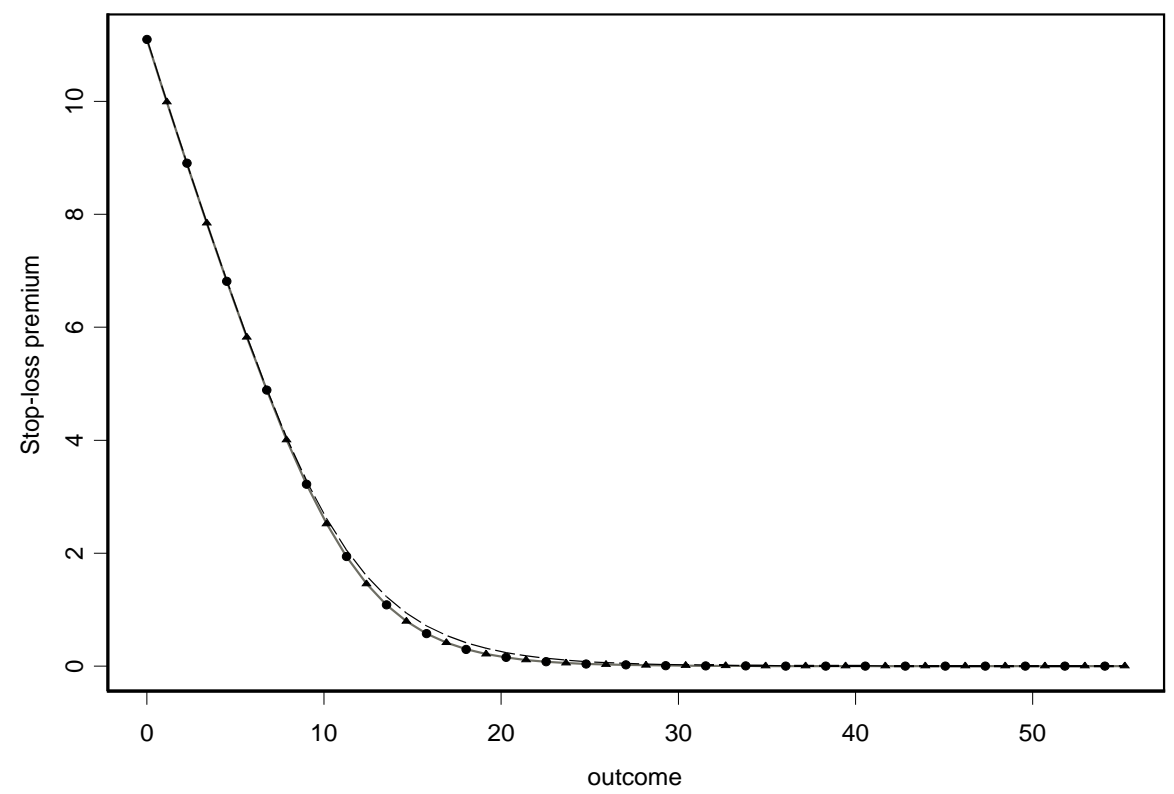

Figure 4: Stop-loss premiums for ' $S_{s p, 65}$ ' (MC) (solid grey line), $S_{s p, 65}^{l}\left(\bullet-\right.$ line), $S_{s p, 65}^{c l}(\mathbf{\Delta}$-line) and $S_{s p, 65}^{c}$ (dashed line).

\subsection{A homogeneous portfolio of life annuities}

In this subsection we consider the distribution of the present value of a homogeneous portfolio of $N_{0}$ life annuities paying a fixed amount 1 of yearly benefit. This present value can be expressed by the formula

$$
S_{p p, x}=\sum_{i=1}^{\lfloor\omega-x\rfloor} N_{i} \alpha_{i} e^{-Y(i)},
$$

where $N_{i}$ denotes the number of survivals in year $i$ and can be written as

$$
N_{i}=I_{\left(T_{x}^{(1)}>i\right)}+I_{\left(T_{x}^{(2)}>i\right)}+\ldots+I_{\left(T_{x}^{\left(N_{0}\right)}>i\right)}
$$

We assume that the random variables $T_{x}^{(j)}$ are mutually independent, where $T_{x}^{(j)}$ denotes the future lifetime of the $j$-th insured. So the random variables $N_{i}$ are binomially distributed with parameters $n=N_{0}$ and success parameter ${ }_{i} p_{x}$.

Note that

$$
S_{p p, x}=\sum_{j=1}^{N_{0}} S_{s p, x}^{(j)},
$$


with $S_{s p, x}^{(j)}$ given by

$$
S_{s p, x}^{(j)}=\sum_{i=1}^{\lfloor\omega-x\rfloor} I_{\left(T_{x}^{(j)}>i\right)} \alpha_{i} e^{-Y(i)} .
$$

The computation of the convex upper and lower bound for the case of a portfolio of life annuities is more complicated than in the case of a single life annuity. The binomial distributed random variables $N_{i}$ are not very useful in practical computations, because there exist no closed-form expressions for the cumulative and the inverse distribution functions. This problem can be dealt with by replacing the random variables $N_{i}$ by more handy continuous approximations $\tilde{N}_{i}$. We propose to approximate the distribution of $N_{i}$ by the Normal Power Approximation (NPA). This allows to incorporate the sknewness in contrast with a Normal approximation, because the binomial distribution is very skewed (unless either the parameter $n$ is very high or the success parameter $p$ is close to $\frac{1}{2}$ ). The distribution function of the NPA $\tilde{N}_{i}$ is given by the formula

$$
F_{\tilde{N}_{i}}(x)=\Phi\left(-\frac{3}{\gamma_{N_{i}}}+\sqrt{\frac{9}{\gamma_{N_{i}}^{2}}+\frac{6\left(x-\mu_{N_{i}}\right)}{\gamma_{N_{i}} \sigma_{N_{i}}}+1}\right),
$$

where

$$
\begin{aligned}
\mu_{N_{i}} & =\mathrm{E}\left[N_{i}\right]=N_{0 i} p_{x} \\
\sigma_{N_{i}}^{2} & =\operatorname{Var}\left[N_{i}\right]=N_{0}{ }_{i} p_{x i} q_{x} \\
\gamma_{N_{i}} & =\frac{\mathrm{E}\left[\left(N_{i}-\mu_{N_{i}}\right)^{3}\right]}{\sigma_{N_{i}}^{3}}=\frac{1-2_{i} p_{x}}{\sqrt{N_{0} p_{x i} q_{x}}} .
\end{aligned}
$$

Then the $p$-th quantile of $\tilde{N}_{i}$ is given by

$$
F_{\tilde{N}_{i}}^{-1}(p)=\mu_{N_{i}}+\sigma_{N_{i}} \Phi^{-1}(p)+\frac{\gamma_{N_{i}} \sigma_{N_{i}}}{6}\left(\left(\Phi^{-1}(p)\right)^{2}-1\right) .
$$

\subsubsection{The upper bound}

The upper bound $S_{p p, x}^{c}$ is computed as described in Section 3.5.1. The only difference is that in the formulae (13), (14) and (17) $F_{X_{i}}^{-1}(u)$ is replaced by the approximation given in (29).

\subsubsection{The lower bound}

To compute the lower bound one has to choose two conditioning variables: $\Gamma$ and $\Lambda$. For the first conditioning random variable $\Gamma$ we propose to take $N_{i_{0}}$ - the number of policies-in-force in the year $i_{0}$. Note that

$$
\mathrm{E}\left[N_{i} \mid N_{i_{0}}=n_{0}\right]={ }_{i-i_{0}} p_{x+i_{0}} n_{0} \quad \text { for } i \geq i_{0} .
$$


For $i<i_{0}, \operatorname{Pr}\left[N_{i}=n \mid N_{i_{0}}=n_{i}\right]$ can be computed from Bayes theorem. As a result one gets the following formula for the conditional expectation:

$$
\begin{aligned}
\mathrm{E}\left[N_{i} \mid N_{i_{0}}=n_{0}\right] & =\sum_{k=n_{0}}^{N_{0}} k \frac{\operatorname{Pr}\left[N_{i_{0}}=n_{0} \mid N_{i}=k\right] \operatorname{Pr}\left[N_{i}=k\right]}{\operatorname{Pr}\left[N_{i_{0}}=n_{0}\right]} \\
& =\sum_{k=n_{0}}^{N_{0}} k \frac{\left(\begin{array}{c}
k \\
n_{0}
\end{array}\right)\left(\begin{array}{c}
N_{0} \\
k
\end{array}\right)}{\left(\begin{array}{c}
N_{0} \\
n_{0}
\end{array}\right)} \frac{{ }_{i_{0}-i} p_{x+i}^{n_{0}} i_{0}-i q_{x+i}^{k-n_{0}}{ }_{i} p_{x}^{k}{ }_{i} q_{x}^{N_{0}-k}}{i_{0} p_{x}^{n_{0}}{ }_{i} q_{x}^{N_{0}-n_{0}}} \\
& =\sum_{k=n_{0}}^{N_{0}} k\left(\begin{array}{c}
N_{0}-n_{0} \\
k-n_{0}
\end{array}\right){ }_{i} p_{x}^{k-n_{0}} \frac{i_{0}-i q_{x+i}^{k-n_{0}} q_{x}^{N_{0}-k}}{i_{0} q_{x}^{N_{0}-n_{0}}} .
\end{aligned}
$$

For mathematical convenience we rewrite this formula for non-integer values of $N_{i_{0}}$ as follows

$$
\mathrm{E}\left[N_{i} \mid N_{i_{0}}=y\right]=\sum_{k=\lceil y\rceil}^{N_{0}} k\left(\begin{array}{c}
N_{0}-\lceil y\rceil \\
k-\lceil y\rceil
\end{array}\right) i p_{x}^{k-\lceil y\rceil} \frac{i_{0}-i q_{x+i}^{k-\lceil y\rceil} i q_{x}^{N_{0}-k}}{i_{0} q_{x}^{N_{0}-\lceil y\rceil}},
$$

where $\lceil$.$\rceil is the ceiling function, i.e. \lceil x\rceil$ is the smallest integer greater than or equal to $x$.

We propose to take $\Lambda^{(a)}$, as defined in Section 4.1.2, for the second conditioning random variable $\Lambda$. Now one can perform step by step the computations described in Section 3.5.2 with the only exception that $\mathrm{E}\left[X_{i} \mid \Gamma=\gamma\right]$ has to replaced in the formulas (21) and (22) by $\mathrm{E}\left[N_{i} \mid N_{i_{0}}=y\right]$ in (30).

Also the stop-loss premiums are calculated according to the methodology presented in Section 5.3 and 3.5.2 with the only difference the replacement of $\mathrm{E}\left[X_{i} \mid \Gamma=F_{\Gamma}^{-1}(u)\right]$ in formula (24) by the appoximation given in (30).

\subsubsection{The moments based approximation}

As in the case of a single life annuity, the only problem in the computation of the weight $z$ given by (9) is to find expressions for the variances of $S_{p p, x}^{c}, S_{p p, x}^{l}$ and $S_{p p, x}$. For the upper and the lower bound we have deployed a procedure, described in Section 3.5.3, with $f_{i}(u)$ replaced by

$$
f_{i}(u)=F_{\tilde{N}_{i}}^{-1}(u) \text { for the upper bound }
$$

and

$$
f_{i}(u)=\mathrm{E}\left[N_{i} \mid N_{i_{0}}=F_{\tilde{N}_{i_{0}}}^{-1}(u)\right] \text { for the lower bound. }
$$

The variance of $S_{p p, x}$ can be computed from (28) and by noticing that the random variables $\left\{S_{s p, x}^{(j)}\right\}_{j}$ are, given the returns $\vec{Y}=(Y(1), Y(2), \ldots, Y(\lfloor\omega-x\rfloor))$, conditionally independent. Hence, we have that

$$
\begin{aligned}
\operatorname{Var}\left[S_{p p, x}\right] & =\mathrm{E}\left[\operatorname{Var}\left[S_{p p, x} \mid \vec{Y}\right]\right]+\operatorname{Var}\left[\mathrm{E}\left[S_{p p, x} \mid \vec{Y}\right]\right] \\
& =N_{0} \mathrm{E}\left[\operatorname{Var}\left[S_{s p, x} \mid \vec{Y}\right]\right]+N_{0}^{2} \operatorname{Var}\left[\mathrm{E}\left[S_{s p, x} \mid \vec{Y}\right]\right] \\
& =N_{0} \operatorname{Var}\left[S_{s p, x}\right]+\left(N_{0}^{2}-N_{0}\right) \operatorname{Var}\left[\mathrm{E}\left[S_{s p, x} \mid \vec{Y}\right]\right]
\end{aligned}
$$


where $\operatorname{Var}\left[S_{s p, x}\right]$ is calculated in Section 4.1 .4 and

$$
\operatorname{Var}\left[\mathrm{E}\left[S_{s p, x} \mid \vec{Y}\right]\right]=\sum_{i=1}^{\lfloor\omega-x\rfloor} \sum_{j=1}^{\lfloor\omega-x\rfloor}{ }_{i} p_{x}{ }_{j} p_{x} \alpha_{i} \alpha_{j} e^{-\mu_{i}-\mu_{j}+\frac{\sigma_{i}^{2}+\sigma_{j}^{2}}{2}}\left(e^{\sigma_{i j}}-1\right) .
$$

\subsubsection{A numerical illustration}

To test the quality of the derived approximations we present a numerical illustration similar to this from Section 4.1.5. As before we work in a Black \& Scholes setting with drift $\mu=0.05$ and volatility $\sigma=0.1$ and we use the Makeham-Gompertz law to describe the mortality process of 65 -year old male persons. We compare the performance of the lower bound $S_{p p, 65}^{l}$, the upper bound $S_{p p, 65}^{c}$ and the moments based approximation $S_{p p, 65}^{m}$ with the real value $S_{p p, 65}$, obtained by extensive simulation, for a portfolio of 1000 policies. The number of policies-in-force after the first year $N_{1}$ is taken as conditioning random variable $\Gamma$ for the lower bound. This choice seems to us to be reasonable - other choices can improve the performance of the lower bound only a bit but with a significant increase in computational time as cost. The Monte Carlo (MC) study of $S_{p p, 65}$ is based on $30 \times 50000$ simulated paths. Antithetic variables are used in order to reduce the variance of the Monte-Carlo estimates.

The quality of the approximations is illustrated in Figure 5 and 6 . One can see that the lower bound $S_{p p, 65}^{l}$ indeed performs very good. The fit of the upper bound is a bit poorer but still reasonable. The moments based approximation $S_{p p, 65}^{m}$ performs extremely good. These visual observations are confirmed by the numerical values of some upper quantiles displayed in Table 4 and by the study of stop-loss premiums in Figure 7 and in Table 5.

\begin{tabular}{lllll}
\hline$p$ & $S_{p p, 65}^{l}$ & $S_{p p, 65}^{m}$ & $S_{p p, 65}^{c}$ & $\mathrm{MC}$ (s.e.) \\
\hline 0.995 & 20209 & 20250 & 22620 & $20242(22.09)$ \\
0.975 & 17252 & 17272 & 18722 & $17276(8.80)$ \\
0.95 & 15937 & 15951 & 17029 & $15947(8.15)$ \\
0.90 & 14565 & 14574 & 15290 & $14568(5.08)$ \\
0.75 & 12574 & 12577 & 12821 & $12577(3.90)$ \\
\hline
\end{tabular}

Table 4: Approximations for some selected quantiles with probability level $p$ of $S_{p p, 65}$.

\begin{tabular}{lllll}
\hline$d$ & $S_{p p, 65}^{l}$ & $S_{p p, 65}^{m}$ & $S_{p p, 65}^{c}$ & MC (s.e.) \\
\hline 0 & 11094 & 11094 & 11094 & $11098(2.11)$ \\
5000 & 6094 & 6094 & 6095 & $6098(2.10)$ \\
10000 & 1608 & 1610 & 1793 & $1611(1.95)$ \\
15000 & 153.7 & 155.3 & 278.4 & $155.3(1.78)$ \\
20000 & 10.23 & 10.57 & 36.02 & $10.67(1.26)$ \\
25000 & 0.680 & 0.734 & 4.816 & $0.743(0.09)$ \\
30000 & 0.051 & 0.059 & 0.711 & $0.036(0.02)$ \\
\hline
\end{tabular}

Table 5: Approximations for some selected stop-loss premia with retention $d$ of $S_{p p, 65}$. 


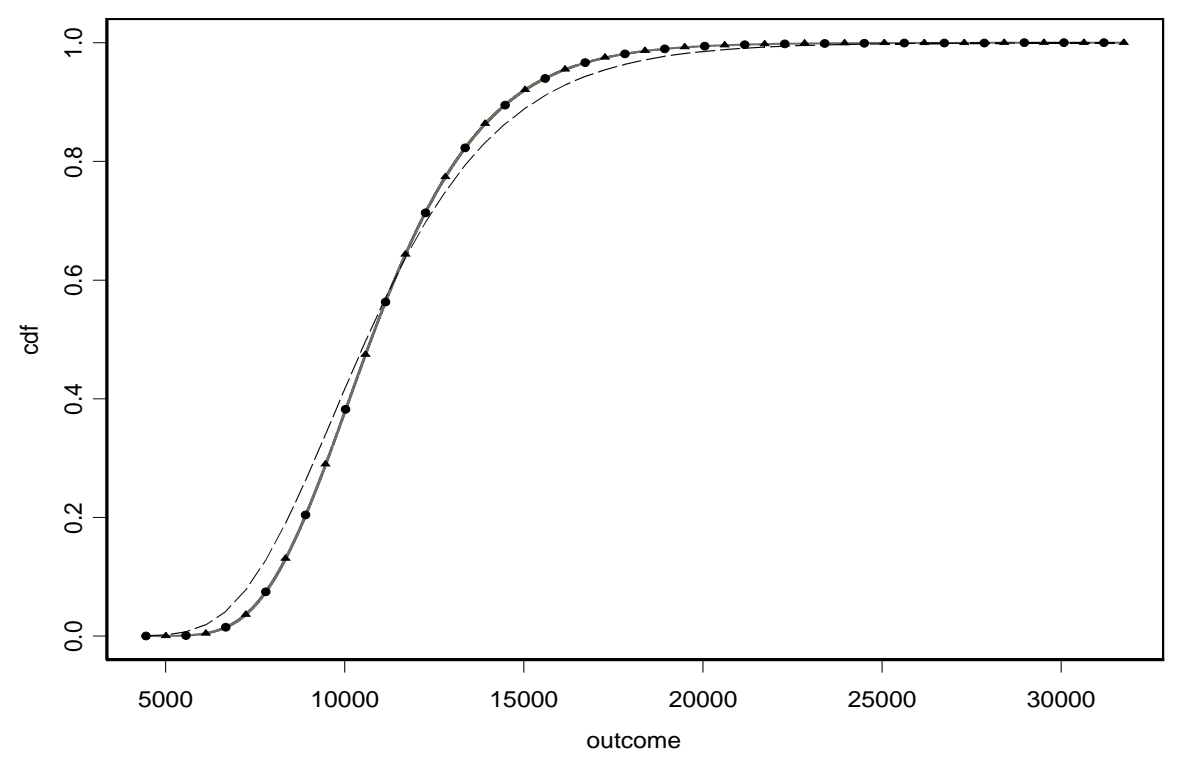

Figure 5: The cdf's of ' $S_{p p, 65}$ ' (MC) (solid grey line), $S_{p p, 65}^{l}\left(\bullet-\right.$ line), $S_{p p, 65}^{m}$ (८-line) and $S_{p p, 65}^{c}$ (dashed line).

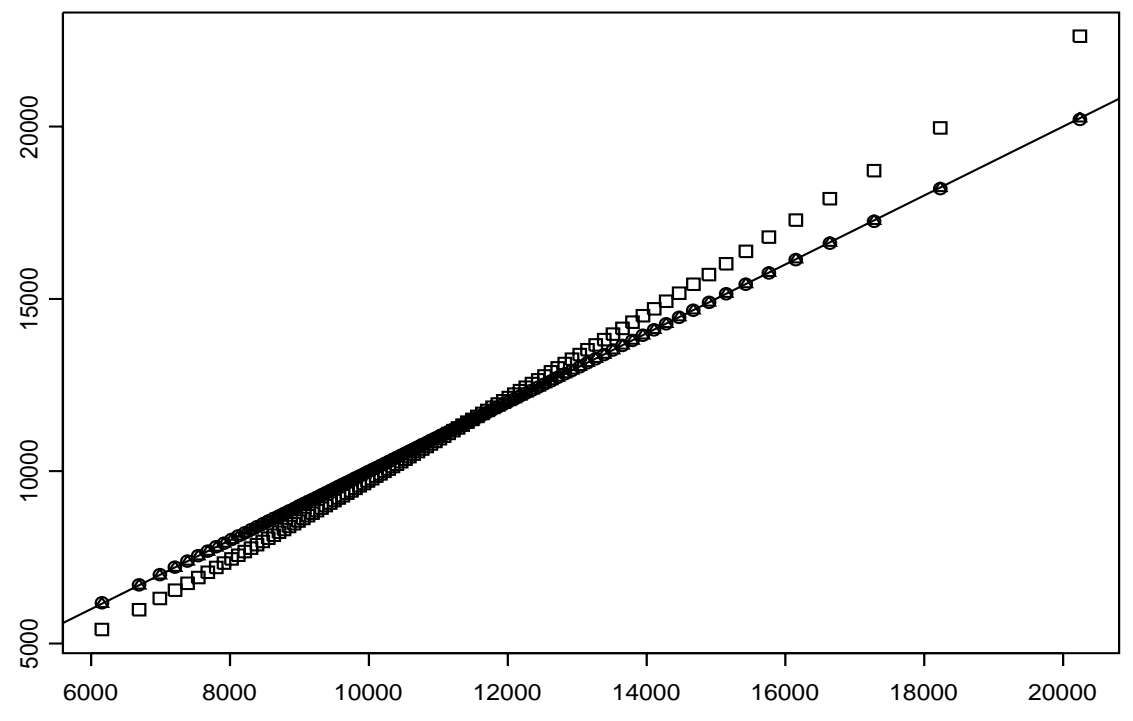

Figure 6: QQ-plot of the quantiles of $S_{p p, 65}^{l}(\circ), S_{p p, 65}^{m}(\triangle)$ and $S_{p p, 65}^{c}(\square)$ versus those of ' $S_{p p, 65}$ ' (MC). 


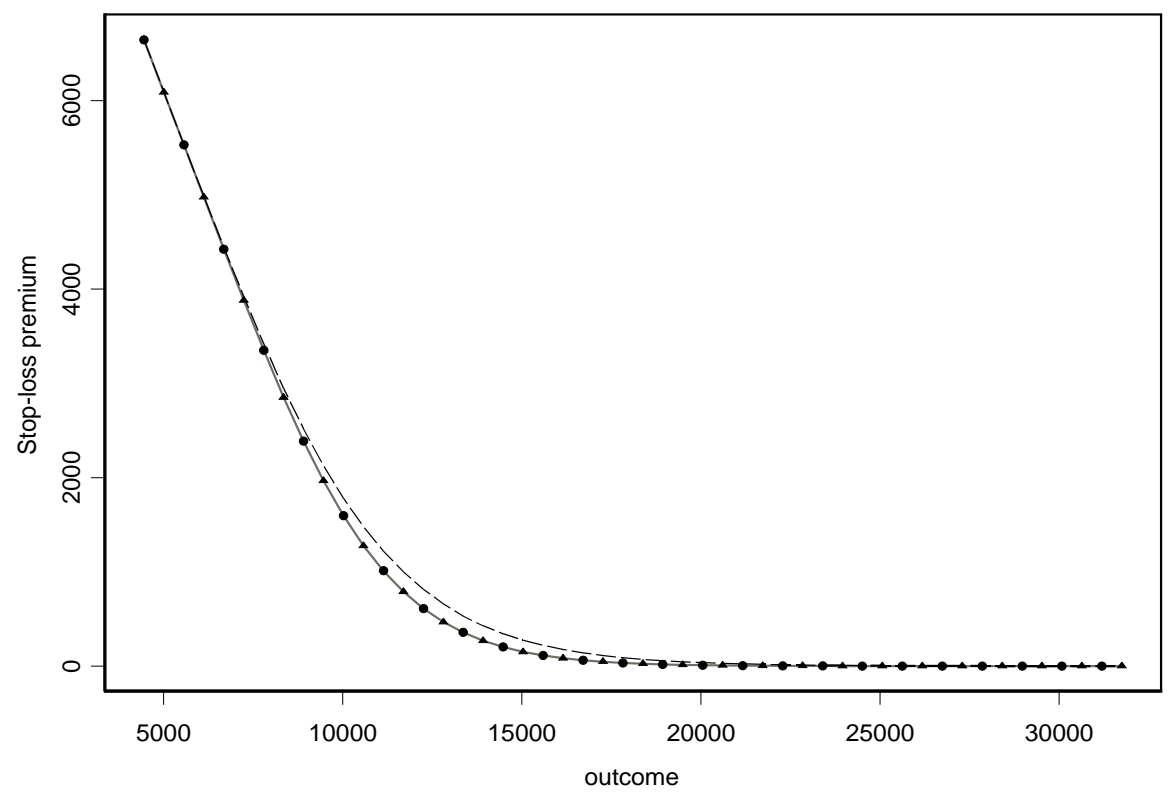

Figure 7: Stop-loss premiums for ' $S_{p p, 65}$ ' (MC) (solid grey line), $S_{p p, 65}^{l}(\bullet-l i n e), S_{p p, 65}^{m}$ (ム-line) and $S_{p p, 65}^{c}$ (dashed line).

\section{$5 \quad$ Summary and conclusions}

The random nature of interest rates causes serious problems in determinig fair values of actuarial reserves. In such a case the aggregate distribution function of future cash-flows is very difficult to estimate. Usually it is only possible to get formulae for the first couple of moments. To compute more cumbersome risk measures, like stop-loss premiums or upper quantiles, one has to rely on time consuming simulations.

In this contribution we proposed an alternative solution for the case of life annuities. We derived comonotonicity-based approximations both for the case of a single life annuity and a homogeneous portfolio of life annuities. The numerical illustrations confirm the very high accuracy of the bounds (especially the lower bound). These observations are confirmed by the results of the stop-loss premia.

One maybe get an impression that the upper bound - which performs poorer than the lower bound in all cases - is not worth being studied. In actuarial applications however the upper bound should draw a lot of attention because one is usually interested in conservative estimates of quantities of interest. Indeed, when an actuary calculates reserves he has to take into account some additional sources of uncertainty, such as the choice of the interest rates model, the estimation of parameters, the assumptions about mortality, the longevity risk and many others. For these the estimates provided by the upper bound can be in many cases more appropriate 
than the more "accurate" approximations obtained from the lower bound.

Many questions remain still open, for example how to incorporate the longevity risk into the model. This could be a research object for a next paper.

\section{Acknowledgements}

Tom Hoedemakers, Grzegorz Darkiewicz and Marc Goovaerts acknowledge the financial support of the Onderzoeksfonds K.U.Leuven (GOA/02: Actuariële, financiële en statistische aspecten van afhankelijkheden in verzekerings- en financiële portefeuilles).

\section{References}

[1] Ahcan, A., Darkiewicz, G., Dhaene, J., Goovaerts, M.J. and Hoedemakers, T. (2004). Optimal Portfolio Selection: Applications in Insurance Business. Proceedings of the Eighth International Congress on Insurance: Mathematics and Economics, Rome.

[2] Arnold, L. (1974). Stochastic Differential Equations: Theory and Applications, John Wiley \& Sons, New York, 228 pp.

[3] Beekman, J.A. and Fuelling, C.P. (1990). Interest and mortality randomness in some annuities. Insurance: Mathematics \& Economics, 9(2-3), 185-196.

[4] Beekman, J.A. and Fuelling, C.P. (1991). Extra randomness in certain annuity models. Insurance: Mathematics 65 Economics, 10(4), 275-287.

[5] Beekman, J.A. and Fuelling, C.P. (1993). One approach to dual randomness in life insurance. Scandinavian Actuarial Journal, 76(2), 173-182.

[6] Bellhouse, D.R. and Panjer, H.H. (1981). Stochastic Modelling of Interest Rates with Applications to Life Contingencies - Part II. Journal of Risk and Insurance, 48(4), 628-637.

[7] Boyle, P.P. (1976). Rates of Return As Random Variables. Journal of Risk and Insurance, 43(4), 693-711.

[8] Bowers, N.L., Gerber, H.U., Hickman, J.C., Jones, D.A. and Nesbitt, C.J. (1986). Actuarial mathematics. Schaumburg, Ill.: Society of Actuaries.

[9] Cesari, R. and Cremonini, D. (2003). Benchmarking, portfolio insurance and technical analysis: a Monte Carlo comparison of dynamic strategies of asset allocation. Journal of Economic Dynamics and Control, 27, 987-1011.

[10] Dębicka, J. (2003). Moments of the cash value of future payment streams arising from life insurance contracts. Insurance: Mathematics \& Economics, 33(3), 533-550.

[11] Devroye, L. (1986). Non-Uniform random variate generation. Springer-Verlag, New York.

[12] Dhaene, J. (1989). Stochastic interest rates and Autoregressive Integrated Moving Average Processes. ASTIN Bulletin, 19(2), 131-138. 
[13] Dhaene, J. (1990). Distributions in life insurance. ASTIN Bulletin, 20(1), 81-92.

[14] Dhaene, J., Denuit, M., Goovaerts, M., Kaas, R. and Vyncke, D. (2002a). The concept of comonotonicity in actuarial science and finance: theory. Insurance: Mathematics 83 Economics, 31(1), 3-33.

[15] Dhaene, J., Denuit, M., Goovaerts, M., Kaas, R. and Vyncke, D. (2002b). The concept of comonotonicity in actuarial science and finance: applications. Insurance: Mathematics $\mathbb{E}$ Economics, 31(2), 133-161.

[16] Frees, E. (1990). Stochastic life contingencies with solvency considerations. Transactions of the Society of Actuaries, 42, 91-129.

[17] Hoedemakers, T., Beirlant, J., Goovaerts, M. and Dhaene, J. (2003). Confidence bounds for discounted loss reserves. Insurance: Mathematics $\&$ Economics, 33(2), 297-316.

[18] Hoedemakers, T., Beirlant, J., Goovaerts, M. and Dhaene, J. (2005). On the Distribution of Discounted Loss Reserves Using Generalized Linear Models. Scandinavian Actuarial Journal. to be published.

[19] Kaas, R., Dhaene, J. and Goovaerts, M. (2000). Upper and lower bounds for sums of random variables. Insurance: Mathematics 85 Economics, 27(2),151-168.

[20] Kaas, R., Goovaerts, M.J., Dhaene, J. and Denuit, M. (2001). Modern Actuarial Risk Theory. Kluwer Academic, The Netherlands.

[21] Norberg, R. (1990). Payment measures, interest and discounting. An axiomatic approach with applications to insurance. Scandinavian Actuarial Journal, 73, 14-33.

[22] Norberg, R. (1993). A solvency study in life insurance. Proceedings of the Third AFIR International Colloquium, Rome, 822-830.

[23] Panjer, H.H. and Bellhouse, D.R. (1980). Stochastic Modelling of Interest Rates with Applications to Life Contingencies. Journal of Risk and Insurance, 47, 91-110.

[24] Parker, G. (1994a). Moments of the present value of a portfolio of policies. Scandinavian Actuarial Journal, 77(1), 53-67.

[25] Parker, G. (1994b). Stochastic analysis of portfolio of endowment insurance policies. Scandinavian Actuarial Journal, 77(2), 119-130.

[26] Parker, G. (1994c). Limiting distribution of the present value of a portfolio. ASTIN Bulletin, 24(1), 47-60.

[27] Parker, G. (1994d). Two stochastic approaches for discounting actuarial functions. ASTIN Bulletin, 24(2), 167-181.

[28] Parker, G. (1996). A portfolio of endowment policies and its limiting distribution. ASTIN Bulletin, 26(1), 25-33.

[29] Parker, G. (1997). Stochastic analysis of the interaction between investment and insurance risks. North American Actuarial Journal, 1(2), 55-71. 
[30] Schoutens, W. (2003). Lévy Processes in Finance: Pricing Financial Derivatives. Wiley, pp. 170.

[31] Vanduffel, S., Hoedemakers, T. and Dhaene, J. (2004). Comparing approximations for risk measures of sums of non-independent lognormal random variables. Research Report OR 0418, Department of Applied Economics, K.U.Leuven, pp. 13.

[32] Vyncke, D., Goovaerts, M.J. and Dhaene, J. (2004). An accurate analytical approximation for the price of a European-style arithmetic Asian option. Finance, 25, 121-139.

[33] Waters, H.R. (1978). The Moments and Distributions of Actuarial Functions. Journal of the Institute of Actuaries, 105, 61-75.

[34] Wilkie, A.D. (1976). The Rate of Interest as a Stochastic Process: Theory and Applications. Proceedings of the 20th International Congress of Actuaries, Tokyo 1, 325-337.

[35] Wolthuis, H. and Van Hoek, I. (1986). Stochastic Models for Life Contingencies. Insurance: Mathematics \& Economics, 5(3), 217-54.

[36] Yaari, M.E. (1987). The dual theory of choice under risk. Econometrica, 55, 95-115. 